\title{
A relaxation method for the energy and morphology of grain boundaries and interfaces
}

\author{
Brandon Runnels ${ }^{\mathrm{a}}$, Irene J. Beyerlein ${ }^{\mathrm{b}}$, Sergio Conti $^{\mathrm{c}}$, Michael Ortiz ${ }^{\mathrm{a}, *}$ \\ ${ }^{a}$ Division of Engineering and Applied Science, California Institute of Technology, 1200 E California Blvd, Pasadena, \\ California, United States 91125 \\ ${ }^{b}$ Theoretical Division, Los Alamos National Laboratory, P.O. Box 1663, Los Alamos, New Mexico, United States 87545 \\ ${ }^{c}$ Institut für Angewandte Mathematik, Universität Bonn, 53115 Bonn, Germany
}

\begin{abstract}
The energy density of crystal interfaces exhibits a characteristic 'cusp' structure that renders it non-convex. Furthermore, crystal interfaces are often observed to be faceted, i. e., to be composed of flat facets in alternating directions. In this work, we forge a connection between these two observations by positing that the faceted morphology of crystal interfaces results from energy minimization. Specifically, we posit that the lack of convexity of the interfacial energy density drives the development of finely faceted microstructures and accounts for their geometry and morphology. We formulate the problem as a generalized minimal surface problem couched in a geometric measure-theoretical framework. We then show that the effective, or relaxed, interfacial energy density, with all possible interfacial morphologies accounted for, corresponds to the convexification of the bare or unrelaxed interfacial energy density, and that the requisite convexification can be attained by means of a faceting construction. We validate the approach by means of comparisons with experiment and molecular dynamics simulations including symmetric and asymmetric tilt boundaries in face-centered cubic (FCC) and body-centered cubic (BCC) crystals. By comparison with simulated and experimental data, we show that this simple model interfacial energy combined with a general microstructure construction based on convexification is able to replicate complex interfacial morphologies, including thermally-induced morphological transitions.
\end{abstract}

\section{Introduction}

Interfaces in crystalline materials are of great importance in a broad range of applications. They are a unique type of material defect and are of interest on both the atomic scale and the continuum scale. Observed phenomena influenced by interfaces include deformation twinning [1], thermal instability of nanolayered composites [2], interface sliding under deformation [3], interface mobility [4-9], solidification [10], physical vapor deposition [1], and extreme deformation [11], among others.

Faceted interfaces, or interfaces with a zig-zag structure, are ubiquitous in experimental observation. More than a century ago, Wulff proposed a construction for predicting the equilibrium, or energy-minimizing, shape of a crystalline inclusion given a way of computing the interfacial energy as a function of normal vector [12]. Wulff's construction has since formed the basis for the study of crystalline inclusions (cf., e. g., [13-15]). A key insight into the behavior of interfaces may be attributed to Herring, who observed that interfaces whose macroscopic orientation does not coincide with one of the equilibrium shape boundaries tend to form a faceted hill-and-valley structure that is locally energy-minimizing [16]. This propensity of crystal interfaces to equilibrate by forming piecewise-affine interfaces may be understood to be a consequence of the cusp structure of the interfacial energy when regarded as a function of its orientation relative to the abutting crystals. Indeed, crystalline interfaces have a strongly orientation-dependent energy with multiple

${ }^{*}$ Corresponding author 
minima, or cusps, for special orientations. This property causes the corresponding energy functional to lack lower-semicontinuity, i. e., to lack stability with respect to microscopic fluctuations, in turn resulting in non-attainment of the energy minimum and the formation of fine microstructure (cf., e. g., [17, 18]).

This interpretation connects the theory of faceted structures to a variety of other nonconvex minimization problems in mechanics [19] and has led to a number of criteria for energy-minimal facet patterns, including Cahn's vectorized energy formalism for formulating a facet-optimization problem [13], Taylor's formulation of the problem in terms of convexity [14], the use of varifolds to represent infinitely-finely corrugated surfaces with a macroscopic shape [20-22], a common-tangent construction [23] and standard convexification with the problem posed in terms of graphs [24], among others. A quantitative estimate of the distance of low-energy states from the ideal Wulff shape was recently obtained by Figalli and Maggi [25]. The corresponding timeevolution problem has been the object of extensive mathematical study, cf., e. g., [26] and references therein. Further to these mathematical developments, materials scientists have also suggested that the energy of faceted interfaces can be estimated by rotational interpolation between the facet planes or by means of a lever-type rule $[27,28]$.

In the past, the relaxation interpretation of faceting has been presented mainly as a conceptual explanation of the phenomenon of interfacial faceting. Thus, relaxation theory posits the existence of a function $\gamma$ giving the interfacial energy for all possible orientations of the interface. However, in practice the complete function $\gamma$ is not known explicitly for specific materials, which hampers the practical application of relaxation methods. Many partial interface models exist. For instance, Read and Shockley computed interface energy by geometrically determining the misfit dislocation density at an interface [29]. Van der Merwe applied the Peierls and Nabarro model for dislocations to interfaces [30]. Frank and Bilby determined a method for computing the dislocation density at an interface from which the interface energy is obtained [31, 32]. Other models include those of Bollmann [33], Fletcher [34], Li [35] and Bulatov [36], among others. Despite these developments, there has been a paucity of analytical and validated five degree-of-freedom models for arbitrary interfaces that can be taken as a basis for relaxation. In previous work by the authors [37], a geometry-based model of interfacial energy, to be referred to as the covariance model, has been developed. The covariance model has been shown to account for the locations and magnitude of the interface energy minima, or cusp structure, in symmetric tilt and twist grain boundaries (GBs), as well as the general form of the interface energy surface.

The main contribution of the present work is twofold. Firstly, we present a faceting construction for relaxing non-convex interfacial energies. Secondly, we demonstrate, by way of comparison with experiment and molecular dynamics (MD) simulations, the ability of the faceting construction, in conjunction with the covariance model, to predict the equilibrium morphology of crystal interfaces, including symmetric tilt grain boundaries in face-centered cubic (FCC) and body-centered cubic (BCC) crystals. We then present some examples of the model applied to FCC asymmetric tilt grain boundaries, and demonstrate that the approach predicts the relaxed energy and, where experimental observations are available, the morphology of the interfaces. Furthermore, we show that the faceting construction accurately predicts the experimentally observed temperature dependence of FCC asymmetric tilt grain boundaries, including a morphological transition occurring at a critical temperature. 


\section{General setting and relaxation of surface energies}

We begin by formulating the interface-relaxation problem in precise mathematical terms for purposes of subsequent analysis. To this end, it is essential to formulate the theory within an appropriate geometric measure-theoretical framework. Pertinent notation and background may be found in [38].

\subsection{Background}

We assume that the orientation relationship between the two materials is fixed so that the interface energy per unit area depends only on the local orientation of the interface. We parameterize the orientation by the normal $\boldsymbol{\nu} \in S^{2}$ and assume that an interfacial energy $\gamma: S^{2} \rightarrow(0, \infty)$ is given. Here and below, $S^{2}$ denotes the unit sphere in $\mathbb{R}^{3}$. We work in an open domain $\Omega \subseteq \mathbb{R}^{3}$, possibly $\Omega=\mathbb{R}^{3}$, and let $\Omega^{b} \subset \Omega$ be the domain occupied by one crystal and $\Omega^{w}=\Omega \backslash \Omega^{b}$ the domain occupied by the other. We assume $\Omega^{b}$ to be a set of locally finite perimeter in $\Omega$. Given the surface energy $\gamma: S^{2} \rightarrow(0, \infty)$ we define the interfacial energy as

$$
\Gamma\left(\Omega^{b}, \Omega\right):=\int_{\Omega \cap \partial \Omega^{b}} \gamma\left(\boldsymbol{\nu}_{\partial \Omega^{b}}(\boldsymbol{x})\right) d \mathcal{H}^{2}(\boldsymbol{x})
$$

where $\boldsymbol{\nu}_{\partial \Omega^{b}}(\boldsymbol{x})$ is the outer normal to $\Omega^{b}$ at the point $\boldsymbol{x} \in \partial \Omega^{b}$ and $d \mathcal{H}^{2}(\boldsymbol{x})$ denotes integration over a surface. If $\Omega=\mathbb{R}^{3}$ and $\Omega^{b}$ is a half-space, this quantity is infinite and must be replaced by an appropriate average over the surface.

Minimization of the energy (1) is a generalized minimal surface problem studied in the field of geometric measure theory. However, solutions of this problem are generally trivial without additional constraints, as the empty set has zero energy. Therefore, additional constraints, e. g., on the volume or on the behavior of the solution at infinity, need to be appended to the problem in general. In particular, the inclusion problem consists of minimizing $\Gamma\left(E, \mathbb{R}^{3}\right)$ over all sets $E \subset \mathbb{R}^{3}$ of a given volume and rectifiable boundary, namely,

$$
\min \left\{\Gamma\left(E, \mathbb{R}^{3}\right): E \subset \mathbb{R}^{3},|E|=V_{0}\right\} .
$$

The minimizing shape $E^{*}$, if it exists, is said to be $\gamma$-minimal. For convex energies, the solution $E^{*}$ to this problem is well-known and is given by the Wulff construction. Convexity is here interpreted in the sense of the one-homogeneous extension, see (12) and (18) below.

In this work, we specifically consider the problem of an asymptotically flat interface, i. e., an interface that is flat at infinity with given normal $\boldsymbol{\nu} \in S^{2}$. More precisely, we seek to minimize $\Gamma\left(E, \mathbb{R}^{3}\right)$ over all sets $E$ coinciding with the half-space $\left\{\boldsymbol{x} \in \mathbb{R}^{3}: \boldsymbol{x} \cdot \boldsymbol{\nu}<0\right\}$ outside a-possibly large-ball, namely

$$
\min \left\{\Gamma(E, B(\mathbf{0}, 2 R)): E \subset \mathbb{R}^{3}, E \backslash B(\mathbf{0}, R)=\{\boldsymbol{x} \cdot \boldsymbol{\nu}<0\} \backslash B(\mathbf{0}, R)\right\} .
$$

In both the inclusion and flat interface cases, the existence of minimizers is not guaranteed in general. Indeed, if $\gamma$ is not convex, then the energy is not lower semicontinuous. Under these conditions, minimizing sequences may develop arbitrarily fine oscillations and fail to converge strongly, as discussed in the next section.

\subsection{Relaxation}

The mathematical theory of relaxation $[17,18]$ allows for the efficient treatment of the energetics of finely oscillating low-energy states without resolving the fine oscillations explicitly in the kinematics. It has been applied to many problems in continuum mechanics, ranging from solid-solid phase transformations to crystal plasticity, and has enabled efficient finite-element computations on macroscopic grids while resolving correctly the energetics of microstructures at arbitrarily fine scales, cf., e. g., [39, 40].

In the present setting, relaxation goes hand-in-hand with the phenomenon of faceting. Thus, consider a situation in which the boundary of the volume $\Omega^{b}$ does not match one of the preferred orientations corresponding to a cusp in the interfacial energy. Then, it may be possible to find a sequence of domains $\omega_{n}^{b}$ that are very close to $\Omega^{b}$, in some appropriate sense, and have much smaller energy. Specifically, the boundary of $\omega_{n}^{b}$ may oscillate about the boundary of $\Omega^{b}$ while making optimal usage of low-energy orientations. The 
length of the boundary of $\omega_{n}^{b}$ is larger than the length of the boundary of $\Omega^{b}$ in general but, depending on the energy density $\gamma$, its energy may be lower (see Figure 1 for an illustration). Therefore the effective energy of the crystal shape $\Omega^{b}$ is not $\Gamma\left(\Omega^{b}, \Omega\right)$ but, instead, the asymptotic value of the sequence $\Gamma\left(\omega_{n}^{b}, \Omega\right)$, with an optimal choice of the sequence $\omega_{n}^{b}$.

With this limiting process in mind, the relaxation of $\Gamma$ is defined as

$$
\Gamma^{\mathrm{rel}}\left(\Omega^{b}, \Omega\right):=\inf \left\{\liminf _{n \rightarrow \infty} \Gamma\left(\omega_{n}, \Omega\right): \omega_{n} \rightarrow \Omega^{b} \text { in } L^{1}(\Omega)\right\} .
$$

Thus, by definition this limit is the smallest energy that can be achieved by considering all possible sequences of domains $\omega_{n}$ which approximate $\Omega^{b}$. The relevant convergence of the domains is $L^{1}(\Omega)$-convergence of the characteristic functions, i. e., $\omega_{n} \rightarrow \Omega^{b}$ if and only if $\left|\Omega \cap \omega_{n} \backslash \Omega^{b}\right|+\left|\Omega \cap \Omega^{b} \backslash \omega_{n}\right| \rightarrow 0$. Taking the constant sequence $\omega_{n}^{b}=\Omega^{b}$ trivially gives $\Gamma^{\mathrm{rel}}\left(\Omega^{b}, \Omega\right) \leq \Gamma\left(\Omega^{b}, \Omega\right)$. If the energy per unit area $\gamma$ is uniformly positive, in the sense that there exists a constant $c>0$ such that $\gamma(\boldsymbol{\nu}) \geq c$ for all $\boldsymbol{\nu} \in S^{2}$, then $\Gamma$ is coercive with respect to the weak $B V$ topology and it follows readily from the direct method of the calculus of variations that $\Gamma^{\mathrm{rel}}$, unlike typical unrelaxed interfacial energies, has at least one minimizer. Thus, relaxation effectively regularizes the generalized minimal surface problem (1) and ensures the existence of energy-minimizing interfaces.

Our principal aim in this work is to determine explicitly the relaxation $\Gamma^{\text {rel }}$. From standard theory (cf., e. g., [38]) it is known that

$$
\Gamma^{\mathrm{rel}}\left(\Omega^{b}, \Omega\right)=\int_{\Omega \cap \partial \Omega^{b}} \gamma^{\mathrm{rel}}\left(\boldsymbol{\nu}_{\partial \Omega^{b}}\right) d \mathcal{H}^{2},
$$

where $\gamma^{\text {rel }}$ is an effective interfacial energy density that solves the following cell problem. Given a direction $\boldsymbol{\nu}$, choose $\boldsymbol{\nu}_{2}$ and $\boldsymbol{\nu}_{3}$ such that $\left\{\boldsymbol{\nu}, \boldsymbol{\nu}_{2}, \boldsymbol{\nu}_{3}\right\}$ forms an orthonormal basis of $\mathbb{R}^{3}$, and let $Q_{\boldsymbol{\nu}}$ be a cube with sides $\boldsymbol{\nu}, \boldsymbol{\nu}_{2}, \boldsymbol{\nu}_{3}$ centered at the origin. Then, for $L>0$,

$$
\begin{aligned}
\gamma^{\mathrm{rel}}(\boldsymbol{\nu}):= & \inf \left\{\frac{1}{L^{2}} \int_{L Q_{\boldsymbol{\nu}} \cap J_{u}} \gamma\left(\boldsymbol{\nu}_{J_{u}}\right) d \mathcal{H}^{2}: u \in S B V_{\mathrm{loc}}\left(\mathbb{R}^{3} ;\{0,1\}\right)\right. \\
& \left.u\left(\boldsymbol{x}+L \boldsymbol{\nu}_{i}\right)=u(\boldsymbol{x}) \text { for } i=2,3, \quad u(\boldsymbol{x})=0 \text { if } \boldsymbol{x} \cdot \boldsymbol{\nu} \leq-\frac{L}{2}, \quad u(\boldsymbol{x})=1 \text { if } \boldsymbol{x} \cdot \boldsymbol{\nu} \geq \frac{L}{2}\right\} .
\end{aligned}
$$

Here $\boldsymbol{\nu}_{J_{u}}$ is the normal to the jump set of $u$, oriented so that $[u]=1$, and the infimum is taken over all patterns thar are $L \boldsymbol{\nu}_{2}$ - and $L \boldsymbol{\nu}_{3}$-periodic and that give $\Omega^{b}$ on one side, and $\Omega^{w}$ on the other. By an appeal to the scaling $\hat{u}(\boldsymbol{x})=u(\boldsymbol{x} L)$, it can check that the expression on the right-hand side does not depend on $L$. Taking $u(\boldsymbol{x})=1$ if $\boldsymbol{x} \cdot \boldsymbol{\nu}>0$ and $u(\boldsymbol{x})=0$ otherwise, it is readily verified that $\gamma^{\text {rel }} \leq \gamma$. Moreover, by convexity, if $\gamma$ is bounded from below then so is $\gamma^{\text {rel }}$.

The energy $\gamma^{\text {rel }}$ is said to be the relaxed interface energy density per unit area or, alternatively, the relaxation of $\gamma$. We note that: i) $\Gamma^{\mathrm{rel}}$ is itself lower-semicontinuous and, therefore, it is its own relaxation; and ii) two different unrelaxed interfacial energy densities $\gamma_{1}, \gamma_{2}$ may have the same relaxation.

\subsection{Faceting construction}

A faceting construction is a special sequence $\omega_{n}^{b}$ in the definition (6) of the relaxation of the interfacial energy density $\gamma$. We begin by considering the case in which the normal is $\boldsymbol{e}_{3}$ and $\omega_{n}^{b}$ can be represented by means of the graph of a function $z_{n}$. We then say that a sequence of Lipschitz functions $\left\{z_{n}\right\}_{n=0}^{\infty}: \mathbb{R}^{2} \rightarrow \mathbb{R}^{\infty}$ is an $m$-facet sequence if it converges pointwise to zero, in the sense that

$$
\lim _{n \rightarrow \infty}\left|z_{n}(\boldsymbol{y})\right|=0, \quad \forall \boldsymbol{y} \in \mathbb{R}^{2}
$$

and there are $m$ vectors $\left\{\boldsymbol{\xi}_{i}\right\}_{i=1}^{m} \subset \mathbb{R}^{2}$ and numbers $\left\{\mu_{i}\right\}_{i=1}^{m} \subset[0, \infty)$ with the following property: For each $n$, there exists $L_{n} \geq 1$ and a partition $\left\{\Omega_{i}^{n}\right\}_{i=1}^{m}$ of $\mathbb{R}^{2}$ such that $z_{n}$ is $\left(0, L_{n}\right)^{2}$-periodic,

$$
\nabla z_{n}(\boldsymbol{y})=\boldsymbol{\xi}_{i}, \quad \text { for } \boldsymbol{y} \in \Omega_{i}^{n} \text { and } i=1 \ldots m,
$$




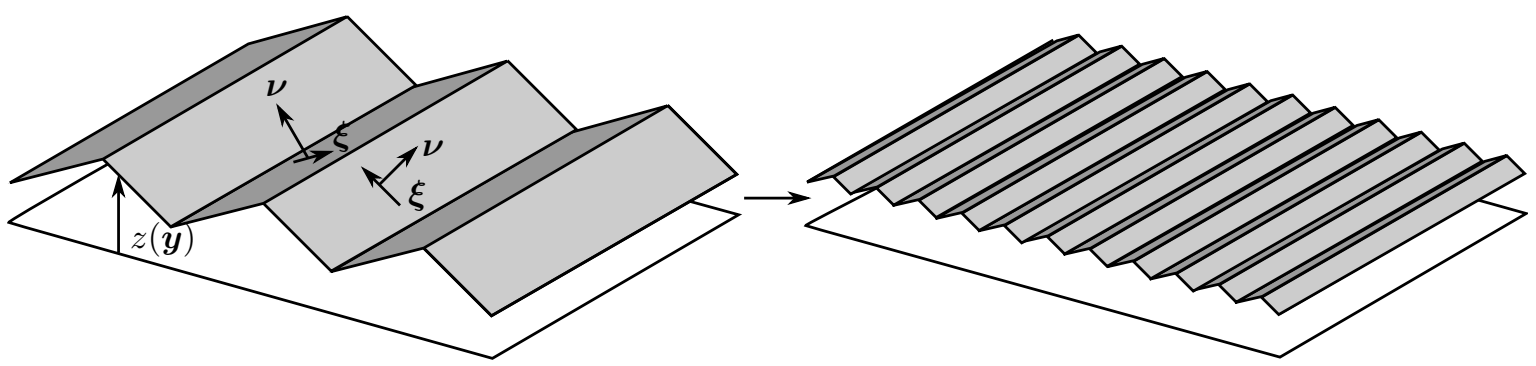

Figure 1: Illustration of the faceting construction: a 2-facet sequence paramaterized by $z_{n}(\boldsymbol{y})$ converges pointwise to zero, whereas the gradient only converges weakly to zero. The vector $\boldsymbol{\xi}$ is the gradient of $z(\boldsymbol{y})$ and $\boldsymbol{\nu}(\boldsymbol{y})$ the normal vector of the facet.

and

$$
\mu_{i}=\lim _{n \rightarrow \infty} \frac{\left|\Omega_{i}^{n} \cap\left(-L_{n} / 2, L_{n} / 2\right)^{2}\right|}{L_{n}^{2}}, \quad \text { for } i=1 \ldots m .
$$

(Figure 1 illustrates the faceting construction with the special case of a 2-facet sequence.) The functions $z_{n}$ are uniformly Lipschitz by $(7 \mathrm{~b})$ and converge locally uniformly to zero by (7a), therefore their gradients converge weakly to zero. In particular, (7b) and (7c) imply that

$$
\sum_{i=1}^{m} \mu_{i} \boldsymbol{\xi}_{i}=\mathbf{0} .
$$

Physically, this may be interpreted as a compatibility condition on the interface that effectively restricts the possible values of $\boldsymbol{\xi}_{i}$ and $\mu_{i}$, and corresponds to the fact that the $m$-facet sequence graph is gapfree. Let $\left\{z_{n}\right\}$ be an $m$-facet sequence with gradients $\left\{\boldsymbol{\xi}_{i}\right\}$ and area fractions $\left\{\mu_{i}\right\}$. After the rescaling $\hat{z}_{n}(\boldsymbol{x}):=z_{n}\left(L_{n} \boldsymbol{x}\right) / L_{n}$, we can assume $L_{n}=1$. Let $\omega_{n}:=\left\{\boldsymbol{x} \in \mathbb{R}^{3}: x_{3}<z_{n}\left(x_{1}, x_{2}\right)\right\}$ be the corresponding domains. Then $\omega_{n} \rightarrow \omega_{*}:=\left\{\boldsymbol{x} \in \mathbb{R}^{3}: x_{3}<0\right\}$, in the sense specified in (4), and, therefore,

$$
\begin{aligned}
\gamma^{\mathrm{rel}}\left(\boldsymbol{e}_{3}\right) & =\Gamma^{\mathrm{rel}}\left(\omega_{*},\left(-\frac{1}{2}, \frac{1}{2}\right)^{3}\right) \leq \lim _{n \rightarrow \infty} \Gamma\left(\omega_{n},\left(-\frac{1}{2}, \frac{1}{2}\right)^{3}\right) \\
& =\lim _{n \rightarrow \infty} \sum_{i=1}^{m} \gamma\left(\boldsymbol{\nu}_{i}\right)\left|\Omega_{i}^{n} \cap\left(-\frac{1}{2}, \frac{1}{2}\right)^{2}\right| \sqrt{1+\left|\boldsymbol{\xi}_{i}\right|^{2}},
\end{aligned}
$$

where $\boldsymbol{\nu}_{i}:=\left(-\boldsymbol{\xi}_{i}, 1\right) / \sqrt{1+\left|\boldsymbol{\xi}_{i}\right|^{2}}$ is the outer normal to $\Omega_{i}^{n}$ and $\left|\Omega_{i}^{n} \cap\left(-\frac{1}{2}, \frac{1}{2}\right)^{2}\right| \sqrt{1+\left|\boldsymbol{\xi}_{i}\right|^{2}}$ is the area of the corresponding part of the interface. Recalling (7c), we obtain

$$
\gamma_{\mathrm{rel}}\left(\boldsymbol{e}_{3}\right) \leq \sum_{i=1}^{m} \mu_{i} \gamma\left(\boldsymbol{\nu}_{i}\right) \sqrt{1+\left|\boldsymbol{\xi}_{i}\right|^{2}} .
$$

We may alternatively extend $\gamma$ positively one-homogeneously to $\mathbb{R}^{3}$ by setting

$$
\bar{\gamma}(\boldsymbol{v}):=|\boldsymbol{v}| \gamma\left(\frac{\boldsymbol{v}}{|\boldsymbol{v}|}\right), \quad \text { for } \boldsymbol{v} \neq \mathbf{0} ; \quad \bar{\gamma}(\mathbf{0}):=0 .
$$

Then, (11) can be more simply written in terms of the vectors $\boldsymbol{a}_{i}:=\left(-\boldsymbol{\xi}_{i}, 1\right) \in \mathbb{R}^{3}$ as

$$
\gamma_{\mathrm{rel}}\left(\boldsymbol{e}_{3}\right) \leq \sum_{i=1}^{m} \mu_{i} \bar{\gamma}\left(\boldsymbol{a}_{i}\right)
$$

and the compatibility condition (8) becomes

$$
\sum_{i} \mu_{i} \boldsymbol{a}_{i}=\boldsymbol{\nu}
$$


where $\boldsymbol{\nu}=\boldsymbol{e}_{3}$ is the normal to the interface.

We proceed to show that, for any set of $m$ vectors $\mu_{i}$ and $\boldsymbol{a}_{i}$ as in (13), with $\left(\boldsymbol{a}_{i}\right)_{3}=1$ for all $i$, a facet sequence can be constructed with the stated properties.

Lemma 1. Let $m \in \mathbb{N}, \mu_{i}>0$ and $\boldsymbol{a}_{i} \in \mathbb{R}^{3}, i=1, \ldots, m$ such that $\left(\boldsymbol{a}_{i}\right)_{3}=1$ for all $i, \sum_{i} \mu_{i}=1$ and (14) holds, with $\boldsymbol{\nu}=\boldsymbol{e}_{3}$. Then there is an $m$-facet sequence such that $\boldsymbol{a}_{i}=\left(-\boldsymbol{\xi}_{i}, 1\right)$.

Proof. We set $\boldsymbol{\xi}_{i}=\left(-\left(a_{i}\right)_{1},-\left(a_{i}\right)_{2}\right)$, for $i=1, \ldots, m$ and $L_{n}=1$.

For $m=1$ the constant sequence $z_{n}(\boldsymbol{x})=0$ will do. For $m=2$ we set $z_{n}(\boldsymbol{x}):=\frac{1}{n} v(n \boldsymbol{x})$, where $v$ is a one-dimensional oscillatory pattern taking gradients $\boldsymbol{\xi}_{1}$ and $\boldsymbol{\xi}_{2}$ on area fractions $\mu_{1}$ and $\mu_{2}$,

$$
v(\boldsymbol{y}):=\min _{i=1,2}\left(\boldsymbol{y} \cdot \boldsymbol{\xi}_{i}-\left\lfloor\mu_{i} \boldsymbol{y} \cdot \boldsymbol{\xi}_{i}\right\rfloor / \mu_{i}\right) .
$$

We remark that $v(\boldsymbol{y})=0$ whenever $\mu_{1} \boldsymbol{y} \cdot \boldsymbol{\xi}_{1}=-\mu_{2} \boldsymbol{y} \cdot \boldsymbol{\xi}_{2} \in \mathbb{Z}$ and that $v$ is Lipschitz-continuous with $D v \in\left\{\boldsymbol{\xi}_{1}, \boldsymbol{\xi}_{2}\right\}$ almost everywhere (see Figure 1). The assertion thus follows.

Now let $m=3$ and assume that no two of the $\boldsymbol{\xi}_{i}$ are parallel, for otherwise the case reduces to the $m=2$ construction. Set $v(\boldsymbol{y}):=\min _{i=1,2,3}\left(1-\boldsymbol{\xi}_{i} \cdot \boldsymbol{y}\right)$ and $T:=\{\boldsymbol{y}: v(\boldsymbol{y})>0\}$. We note that $T$ is a triangle. Therefore, for any $n$, there are $\lambda_{j} \in(0,1 / n)$ and $\boldsymbol{b}_{j} \in \mathbb{R}^{2}$ such that $\mathbb{R}^{2}$ is, up to a null set, the disjoint union of scaled and translated copies of $T, \mathbb{R}^{2}=N \cup \cup_{j}\left(\lambda_{j} T+\boldsymbol{b}_{j}\right)$. Then the functions defined by $z_{n}\left(\lambda_{j} \boldsymbol{y}+\boldsymbol{b}_{j}\right):=\lambda_{j} v(\boldsymbol{y})$ have the required properties.

For $m \geq 4$, we proceed by induction. Assume that $\left\{\xi_{1}, \xi_{2}, \xi_{3}\right\}$ are such that no two of them are parallel. If this choice is not possible, then all vectors are parallel and the $m=2$ construction will do. Set $\bar{\mu}:=\sum_{i=1}^{3} \mu_{i}$, $\overline{\boldsymbol{\xi}}:=\bar{\mu}^{-1} \sum_{i=1}^{3} \mu_{i} \boldsymbol{\xi}_{i}$ and let $\bar{z}_{n}$ be the construction obtained from the vectors $\left(\overline{\boldsymbol{\xi}}, \boldsymbol{\xi}_{4}, \ldots, \boldsymbol{\xi}_{m}\right)$, with weights $\left(\bar{\mu}, \mu_{4}, \ldots, \mu_{m}\right)$. Let $v$ and $T$ be as in the case $m=3$, using the vectors $\overline{\boldsymbol{\xi}}_{i}:=\boldsymbol{\xi}_{i}-\overline{\boldsymbol{\xi}}$ for $i=1,2,3$, with weights $\bar{\mu}_{i}=\mu_{i} / \bar{\mu}$. Let $\bar{\omega}_{n}:=\left\{\boldsymbol{x} \in \mathbb{R}^{2}: D \bar{z}_{n}(\boldsymbol{x})=\overline{\boldsymbol{\xi}}\right\}$. Then, $\bar{\omega}_{n}$ can be covered, up to a null set, with copies of $T$. The map defined by $z_{n}\left(\lambda_{j} \boldsymbol{y}+\boldsymbol{b}_{j}\right):=\bar{z}_{n}\left(\lambda_{j} \boldsymbol{y}+\boldsymbol{b}_{j}\right)+\lambda_{j} v(\boldsymbol{y})$ in $\bar{\omega}_{n}, z_{n}=\bar{z}_{n}$ elsewhere, has the required properties.

By scaling, a similar statement holds in the case $\left(\boldsymbol{a}_{i}\right)_{3}>0$. In turn, the restriction $\left(\boldsymbol{a}_{i}\right)_{3}>0$ can be removed by directly considering facet sequences defined in terms of polygonal sets $\omega_{n}$, not necessarily subgraphs of functions $z_{n}$. Repeating the same procedure with a generic direction $\boldsymbol{\nu}$ instead of $\boldsymbol{e}_{3}$, we obtain

$$
\gamma_{\text {rel }}(\boldsymbol{\nu}) \leq \gamma_{\text {rel }}^{(m)}(\boldsymbol{\nu}) \text { for all } \boldsymbol{\nu}
$$

where

$$
\gamma_{\text {rel }}^{(m)}(\boldsymbol{\nu}):=\inf \left\{\sum_{i=1}^{m} \mu_{i} \bar{\gamma}\left(\boldsymbol{a}_{i}\right): \mu_{i} \in(0, \infty), \boldsymbol{a}_{i} \in \mathbb{R}^{3} \text { such that } \sum_{i=1}^{m} \mu_{i}=1 \text { and } \sum_{i=1}^{m} \mu_{i} \boldsymbol{a}_{i}=\boldsymbol{\nu}\right\} .
$$

We call $\gamma_{\text {rel }}^{(m)}$ the $m$-relaxation of $\gamma$. By Caratheodory's theorem, if $m$ is larger than the space dimension plus one, then $\gamma_{\text {rel }}^{(m)}$ coincides with the convex envelope $\gamma^{* *}$ of $\bar{\gamma}$, cf., e. g., [41]. By Jensen's inequality, definition (6) yields $\gamma^{* *} \leq \gamma^{\text {rel }}$ and, therefore,

$$
\gamma^{\mathrm{rel}}=\gamma_{\mathrm{rel}}^{(m)}=\gamma^{* *}, \quad \text { for all } m \geq 3 .
$$

This identity establishes a direct connection between the relaxation of interfacial energy densities and their convex envelopes.

The physical interpretation of this result is that facets form as the result of energy minimization and, moreover, that the relaxed energy may be computed by minimization over the relatively manageable space of all $m$-facet sequences. This forms the basis for a faceting algorithm to compute energy minimizing facet configurations and relaxed energies, to be discussed in subsequent sections. 


\section{Model and Implementation}

The faceting construction defined in the foregoing and its connection with convexification form the basis of the comparisons with experiment presented in the sequel. In principle, the faceting construction can be applied to any model interfacial energy density. However, as noted in the introduction, there is a paucity of analytical and validated five degree-of-freedom models for arbitrary interfaces that can be taken as a basis for relaxation. Therefore, we shall illustrate the approach with specific reference to the model proposed in [37], herein referred to as the covariance model, which does possess the requisite geometrical generality.

\subsection{Covariance model for interface energy}

For completeness, we begin with a brief overview of the covariance model of interface energy proposed in [37]. It is a discrete, reference-configuration-free model, inspired by the elasticity model proposed by Luckhaus and Mugnai [42], see also [43]. The central idea is to measure locally the distance between two atomic configurations, regarded as sets of points, by means of an estimate of the energetic cost of deforming one into the other. The leading term can be phrased in terms of a localized mass transportation problem, leading to a local measure of the distance between two configurations of atoms in terms of the $L^{2}$ distance between thermalized density measures. In the interfacial problem, the atomic configurations consist of two lattices $\mathcal{L}^{b}$ and $\mathcal{L}^{w}$, which, in the simplest situation, occupy two half-spaces, $\mathbb{R}_{b}^{3}$ and $\mathbb{R}_{w}^{3}$, with $\boldsymbol{\nu}$ the normal to the common boundary. The energy is then given in general form by

$$
\begin{aligned}
\gamma(\boldsymbol{\nu})=\lim _{L \rightarrow \infty} & \frac{1}{L^{2}} \int_{L Q_{\boldsymbol{\nu}} \cap \mathbb{R}_{b}^{3}}\left(\int_{B_{\lambda}(\boldsymbol{z})}\left|\sum_{\boldsymbol{x} \in \mathcal{L}^{w} \cap \mathbb{R}_{w}^{3}} \psi(\boldsymbol{x}-\boldsymbol{p})-\sum_{\boldsymbol{y} \in \mathcal{L}^{b} \cap \mathbb{R}_{w}^{3}} \psi(\boldsymbol{y}-\boldsymbol{p})\right|^{2} \theta(\boldsymbol{z}-\boldsymbol{p}) d \boldsymbol{p}\right) d \boldsymbol{z} \\
+ & \frac{1}{L^{2}} \int_{L Q_{\nu} \cap \mathbb{R}_{w}^{3}}\left(\int_{B_{\lambda}(\boldsymbol{z})}\left|\sum_{\boldsymbol{x} \in \mathcal{L}^{b} \cap \mathbb{R}_{b}^{3}} \psi(\boldsymbol{x}-\boldsymbol{p})-\sum_{\boldsymbol{y} \in \mathcal{L}^{w} \cap \mathbb{R}_{b}^{3}} \psi(\boldsymbol{y}-\boldsymbol{p})\right|^{2} \theta(\boldsymbol{z}-\boldsymbol{p}) d \boldsymbol{p}\right) d \boldsymbol{z} .
\end{aligned}
$$

where $L Q_{\nu}$ is a cube of side length $L$ centered at the origin, with one face normal to $\boldsymbol{\nu}$, and $\theta$ is a general localization function which vanishes outside a ball of radius $\lambda$. (By virtue of this localization function, all integrals can be localized to a small strip around the interface, as the integrand vanishes outside.) We denote by $B_{\lambda}(\boldsymbol{z})$ a ball of radius $\lambda$ centered at $\boldsymbol{z}$.

The functions $\psi(\boldsymbol{x})$ are thermalization functions. $\psi$ is the probability density function for a single atom centered at the origin at finite temperature. $\psi$ approach Dirac distributions in the limit as $T \rightarrow 0$. In calculations, we specifically choose a Gaussian thermalization function corresponding to each atom oscillating in a quadratic potential. Then the $\psi$ are of the form

$$
\psi(\boldsymbol{x})=\frac{1}{\sigma^{3} \pi^{3 / 2}} e^{-\|\boldsymbol{x}\|^{2} / \sigma^{2}}
$$

where $\sigma^{2}=2 k_{B} T / w_{0}$ and $k_{B}$ is Boltzmann's constant, $T$ is the absolute temperature, and $w_{0}$ is an adjustable parameter.

Physically, each term in (19) can be understood as measuring the difference between the atomic positions as they are and the atomic positions as they would be in a perfect lattice. Precisely, the term $\sum_{\boldsymbol{x} \in \mathcal{L}^{b} \cap \mathbb{R}_{b}^{3}} \psi(\boldsymbol{y}-$ $\boldsymbol{x}$ ) gives the effective density at $\boldsymbol{y}$ of the black atoms (which are located in $\mathbb{R}_{b}^{3}$ ). The term $\sum_{\boldsymbol{x} \in \mathcal{L}^{w} \cap \mathbb{R}_{b}^{3}} \psi(\boldsymbol{y}-\boldsymbol{x})$ gives the effective density at $\boldsymbol{y}$ that would have been generated if the atoms had been sitting on the white lattice (still in the half-space $\mathbb{R}_{b}^{3}$ ). In other words, this is the "virtual" density one would obtain if the white lattice would be continued across the interface. If the two densities would coincide, the two lattices would be identical and there would be no interface, hence no interfacial energy. The interfacial energy in (19) measures the distance between the two densities, in an $L^{2}$ sense. We stress that we are only comparing the densities, in particular there is no reference configuration and the model has the full symmetry of the point groups of the lattices. Its explicit treatment is, however, numerically demanding, therefore we shall resort to a simplified version. 
Table 1: Material parameter values used for materials in all GB tests where $E_{0}$ is a scaling constant, $\alpha$ is the lattice parameter, $\sigma / \alpha$ is a nondimensionalized temperature, and $\varepsilon$ is the numerical parameter for the window function.

\begin{tabular}{cccccc}
\hline & $\mathrm{Cu}(\mathrm{FCC})$ & $\mathrm{Au}(\mathrm{FCC})$ & $\mathrm{Al}(\mathrm{FCC})$ & $\mathrm{Mo}(\mathrm{BCC})$ & $\mathrm{Fe}(\mathrm{BCC})$ \\
\hline$E_{0}\left(J / m^{2}\right)$ & 1.45 & 0.95 & 750 & 2.525 & 1.950 \\
$\sigma / \alpha$ & 0.175 & 0.175 & 0.175 & 0.19 & 0.19 \\
$\varepsilon$ & 0.5 & 0.5 & 0.175 & 0.25 & 0.25 \\
\hline
\end{tabular}

We proceed to simplify (19) as follows. Let

$$
\rho^{b, w}(\boldsymbol{y})=\sum_{\boldsymbol{x} \in \mathcal{L}^{b} \cap \mathbb{R}_{b, w}^{3}} \psi(\boldsymbol{y}-\boldsymbol{x}) .
$$

The functions $\rho$ may be interpreted as a density function for the lattice, and indeed

$$
\langle N\rangle_{\Omega}=\int_{\Omega} \rho(\boldsymbol{x}) d x
$$

gives the expected number of atoms in a set $\Omega \subset \mathbb{R}^{3}$. The densities $\rho^{b}$ and $\rho^{w}$ are periodic and square summable. They are, therefore, amenable to an efficient treatment in terms of Fourier series (this would be more problematic in the zero-temperature limit, where the densities become sums of Diracs at the lattice positions). Substituting the definition for $\rho^{b, w}$ into (19) and expanding the square shows that the leading interaction term is

$$
f \rho^{b}(\boldsymbol{y}) \rho^{w}(\boldsymbol{y}) \theta(\boldsymbol{y}) d \boldsymbol{y} \equiv c\left[\rho^{b}, \rho^{w}\right]
$$

where $c\left[\rho^{b}, \rho^{w}\right]$ is the covariance of the distributions $\rho^{b}, \rho^{w}$. The slashed integral denotes the average taken over one periodic cell. In general, the integral in the direction orthogonal to the interface is approximated using quadrature, effectively reducting (23) to a two-dimensional integral over the interface plane. For the window function, we specifically define the window function in terms of its Fourier transform as

$$
\hat{\theta}(\boldsymbol{k})=e^{-\|\boldsymbol{k}\| / \varepsilon},
$$

where $\varepsilon$ is an adjustable parameter and $\boldsymbol{k}$ is in the reciprocal space. With these definitions, the interface energy density can be expressed in terms of the covariance as

$$
\gamma\left(\rho^{b}, \rho^{w}\right)=E_{0}\left(1-\frac{c\left[\rho^{b}, \rho^{w}\right]}{\sqrt{c_{0}\left[\rho^{b}\right] c_{0}\left[\rho^{w}\right]}}\right)
$$

where $c_{0}[\rho]$ is the optimal value of $c[\rho, \rho]$ under rotational transformations and $E_{0}$ is a multiplicative constant.

We note that the covariance model depends on three material parameters, namely: $E_{0}, \sigma$ and $\varepsilon$. Consistent values for these parameters are used across all subsequent comparisons with experiment and MD simulations. The values of the parameters used in calculations are collected in Table 1. While in principle the parameters may be computed based on macro- or microscopic properties of the material, they were determined here by a fit to the MD data.

\subsection{Projected polar plots of interface energy}

A challenge in understanding the faceting behavior of an interface with a particular macroscopic orientation is to visualize the energy surface for that orientation. The landscape of $\gamma(\boldsymbol{n})$ drastically affects the faceting behavior (and consequently the relaxed energy) of the interface, so it is beneficial to visualize the surface in an intuitive way. In this work we use projected polar plot (P3) visualizations. Figure 2 illustrates 


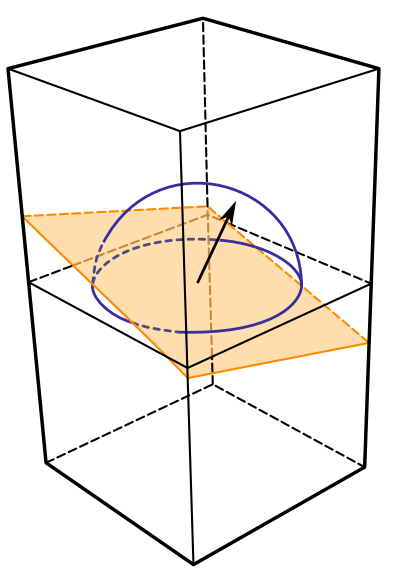

(a) Fixing the orientation relationship between the upper and lower crystals, all remaining interface configurations are determined by normal vector, mapping interface energy to the unit sphere.

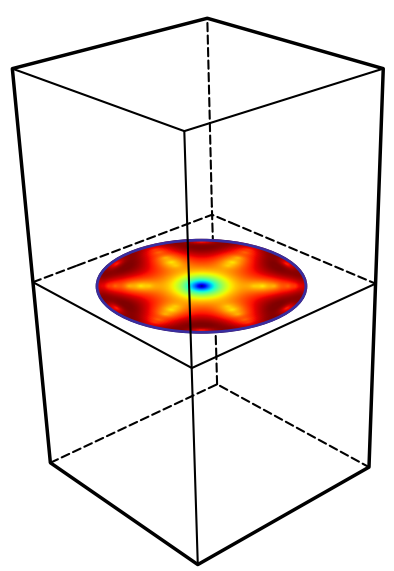

(b) The upper unit 2-hemisphere is homeomorphic to the unit 2-ball over which the energy is plotted. For example, the value at the center corresponds to the normal vector $\boldsymbol{n}=\left[\begin{array}{lll}0 & 0 & 1\end{array}\right]^{T}$.

Figure 2: Computation of a P3 energy plot.

the process for generating P3 visualizations: the orientation relationship is fixed and the interface is allowed to vary. The set of all normal vectors is equivalent to the unit sphere, and therefore it is convenient to associate the energy of each interface inclination with its corresponding point on the unit sphere, generating a plot of energy over the sphere. $\gamma$ is clearly parity invariant, that is, $\gamma(\boldsymbol{n})=\gamma(-\boldsymbol{n})$, indicating that the interface need not be oriented. As a consequence, the unit hemisphere $\left(S_{2}^{+}=\left\{\boldsymbol{x} \in \mathbb{R}^{3}:|\boldsymbol{x}|=1, x_{3} \geq 0\right\}\right)$ is sufficient to capture all of the interface behavior. Because it is homeomorphic to the unit 2-ball, we use the projection $\left(n_{1}, n_{2}, n_{3}\right) \mapsto\left(n_{1}, n_{2}\right)$ to visualize the energy surface.

P3 visualizations provide a convenient means of describing optimal facet patterns. A collection of normal vectors can be visualized as a collection of points, and the resultant facet pattern as a convex polygon connecting them. We see that the constraint in (14) indicates that a set of facet vectors $\{\boldsymbol{n}\}$ form a facet pattern only if their convex hull contains the origin; consequently a polyhedral representation of a facet pattern gives an immediate measure of their compatibility.

\subsection{Procedure}

We implement a basic grid search algorithm to compute the relaxation of $\gamma$. We use the following procedure for each relaxed energy point. First the orientation relationship of the crystals is fixed, so that $\gamma=\gamma(\boldsymbol{n})$. A uniform grid of normal vectors $\left\{\boldsymbol{n}_{i}\right\} \subset \mathcal{S}_{2}^{+}$is generated, and the covariance model is used to compute the energy at each point, giving a set of values $\left\{\lambda_{i}\right\} \subset \mathbb{R}^{+}$.

We refer to " $m$-relaxation" as the relaxation given by solving (14) in the space of $m$-facet sequences; e.g. a 2-relaxation returns the optimal 2 -facet pattern and the corresponding lower energy. Since $m$-facets $\subset$ $(m+1)$-facets, $\gamma_{C}^{m} \geq \gamma_{C}^{m+1}$ with the superscript of $\gamma_{C}$ denoting the order of the relaxation.

Algorithm 1 outlines the general procedure for computing the 3-relaxation; the algorithm for relaxation order 2 is similar. By (18) an order higher than 3 is not necessary. The basic procedure has $\mathcal{O}\left(n^{m}\right)$ time complexity, where $n$ is the number of grid points and $m$ is the order of the faceting. A number of measures can be exploited that improve the efficiency of the computation, such as selective sampling, partitioning of the domain, and numerical annealing. In addition, known symmetries of $\gamma$ can be exploited to significantly 
reduce the search space. However, we find that the basic method does not require extensive improvement in order to return results.

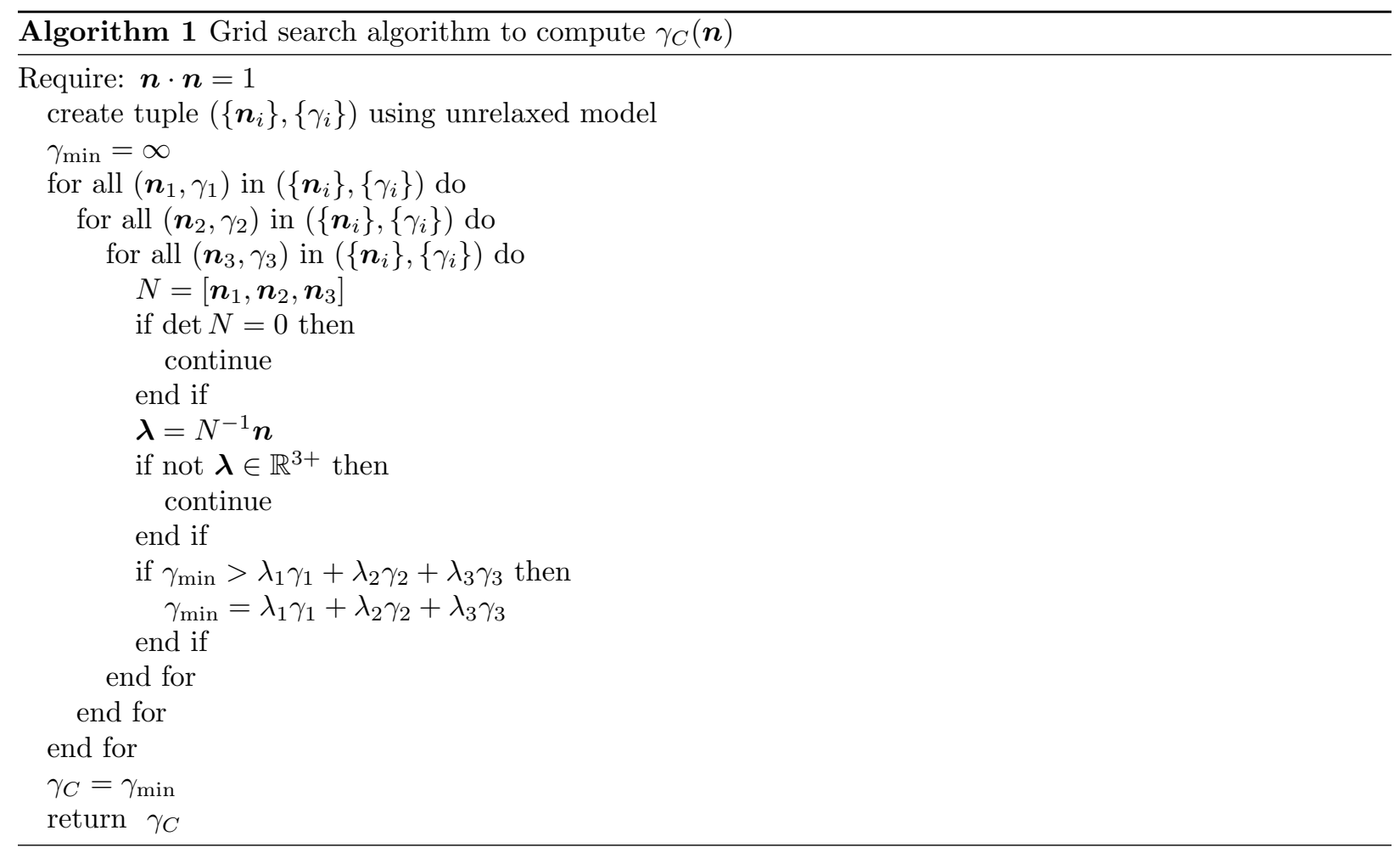

\subsection{Implementation}

The unrelaxed results were generated using the same $\mathrm{C}++$ code as in [37]. For each relaxed material point, the code was used to generate a P3 dataset. Threading was implemented using pthreads and the code was run with 7-8 processes. The computation of each P3 dataset (with high enough resolution for the relaxation method to accuratly find the minimal cusp value) took generally 1-2 seconds, although this number varied slightly depending on the value of the dimensionless temperature. The relaxation method was used on each dataset to compute the optimal faceting pattern. For many cases the 2- and 3-relaxation results were identical, although in some cases it was necessary to run a full 3-relaxation. With datasets large enough to find cusps accurately and with no data reduction, each relaxation computation took approximately 1-2 seconds to complete.

\section{Validation examples}

We proceed to validate the faceting construction, applied to the covariance model of interfacial energy, by comparison with selected experimental observations and MD simulations. We specifically seek to demonstrate the ability of the faceting construction to predict the equilibrium morphology of crystal interfaces, including symmetric and asymmetric tilt boundaries in face-centered cubic (FCC) and body-centered cubic (BCC) crystals, including morphological transitions occurring at a critical temperature.

\subsection{Symmetric tilt grain boundaries}

The set of symmetric tilt grain boundaries (STGBs) forms one of the most basic classes of nontrivial grain boundaries. STGBs are obtained are obtained by beginning with a uniform crystal and rotating the 


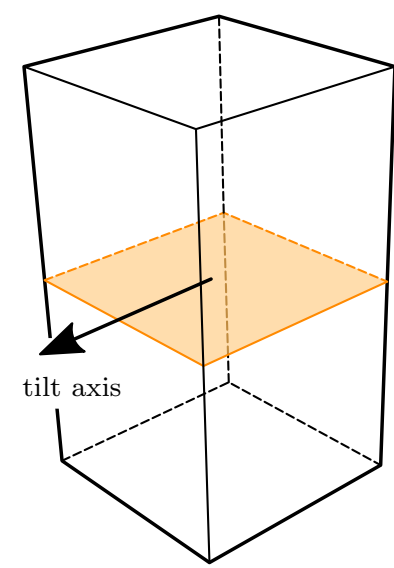

(a) Begin with a uniform crystal

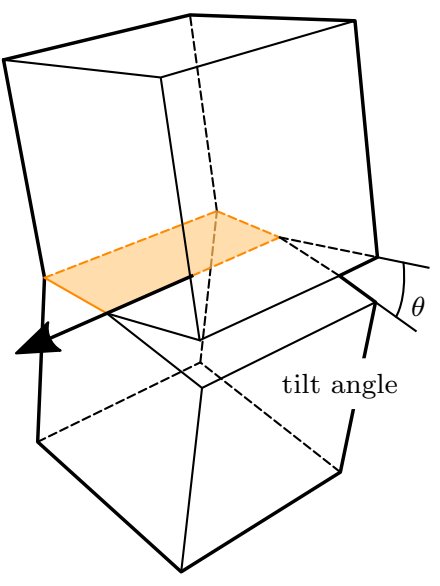

(b) Rotate symmetrically about tilt axis to form STGB

Figure 3: Geometry of symmetric tilt grain boundaries

upper and lower halves symmetrically with respect to a tilt axis contained in the prescribed interface (Figure 3). Consequently, STGBs are defined by i) the crystallographic direction (in Miller indices) corresponding to the tilt axis that remains constant, ii) the total tilt angle between the two crystals about the tilt axis, and iii) the direction corresponding to the initial normal axis to the homogeneous interface. Because STGBs are by definition symmetric about the constant interface plane, the tilt axes must lie within a symmetry plane of the crystal [44]; as such the class of STGBs is limited to a selection of tilt axes. For cubic materials the STGB tilt axes are $\langle 100\rangle,\langle 110\rangle,\langle 111\rangle$, and $\langle 112\rangle$, and we have found a wide selection of MD data for these STGBs $[45,46]$.

The results in this section and in section 4.2 follow the results in the previous work by the authors on the covariance model for interface energy [37]. As discussed in the original formulation, the planar restriction on the covariance model results in overprediction of the energy of some boundaries, particularly for boundaries in homogeneous crystals. For many of the cases described we find that the unrelaxed model suffices and that no change is effected by relaxation. For a thorough discussion of the behavior of the grain boundary energy for the unrelaxed regions we refer the reader to the original analysis. In this work we are concerned with the effect of relaxation; consequently we discuss the improvements on the unrelaxed results only.

In Figure 4 we observe the effect of relaxation on the set of FCC STGBs. The original results for the unrelaxed model are plotted using a finely dashed line and the results for the relaxed model are plotted using a solid line; results for copper and gold are plotted in blue and green, respectively. We note that, in general, the unrelaxed and relaxed results are coincident; relaxation occurs in the regions where they diverge.

For tilts about the [100] axis, relaxation occurs for $0^{\circ} \leq \theta<5^{\circ}$ and $75^{\circ}<\theta \leq 90^{\circ}$. The result for the $0^{\circ} \leq \theta<5^{\circ}$ region corrects a discrepancy in the unrelaxed model; at $\theta=0$ the crystal is uniform and should therefore have zero grain boundary excess energy. The relaxation method effects this energy reduction by creating facets for the $\{111\}$ boundary planes, obtained by rotating the interface about the $\{110\}$ axes. That the resultant energy would be reduced to zero follows by the observation that the $\{111\}$ family of STGBs are energy minimizers; from the [110] tilt plot we see that the unrelaxed energy is zero for the $\{111\}$ case. Consequently we observe that this relaxation construction occurs for all $\{100\}$ symmetric tilt boundaries, (the other occurring for [110] tilt at $175^{\circ}<\theta \leq 180^{\circ}$ ).

For the [110], [111] and [112] tilt boundaries we observe significant relaxation for $0^{\circ} \leq \theta<15^{\circ}$, all of which correspond to the case of near-uniform crystals. As before, by examining the energy plot we see that the relaxation to zero is effected by convexification with $\{111\}$ facets, all of which have zero energy.

In Figure 5 we perform a similar set of tests using materials with BCC crystal structure, molybdenum 

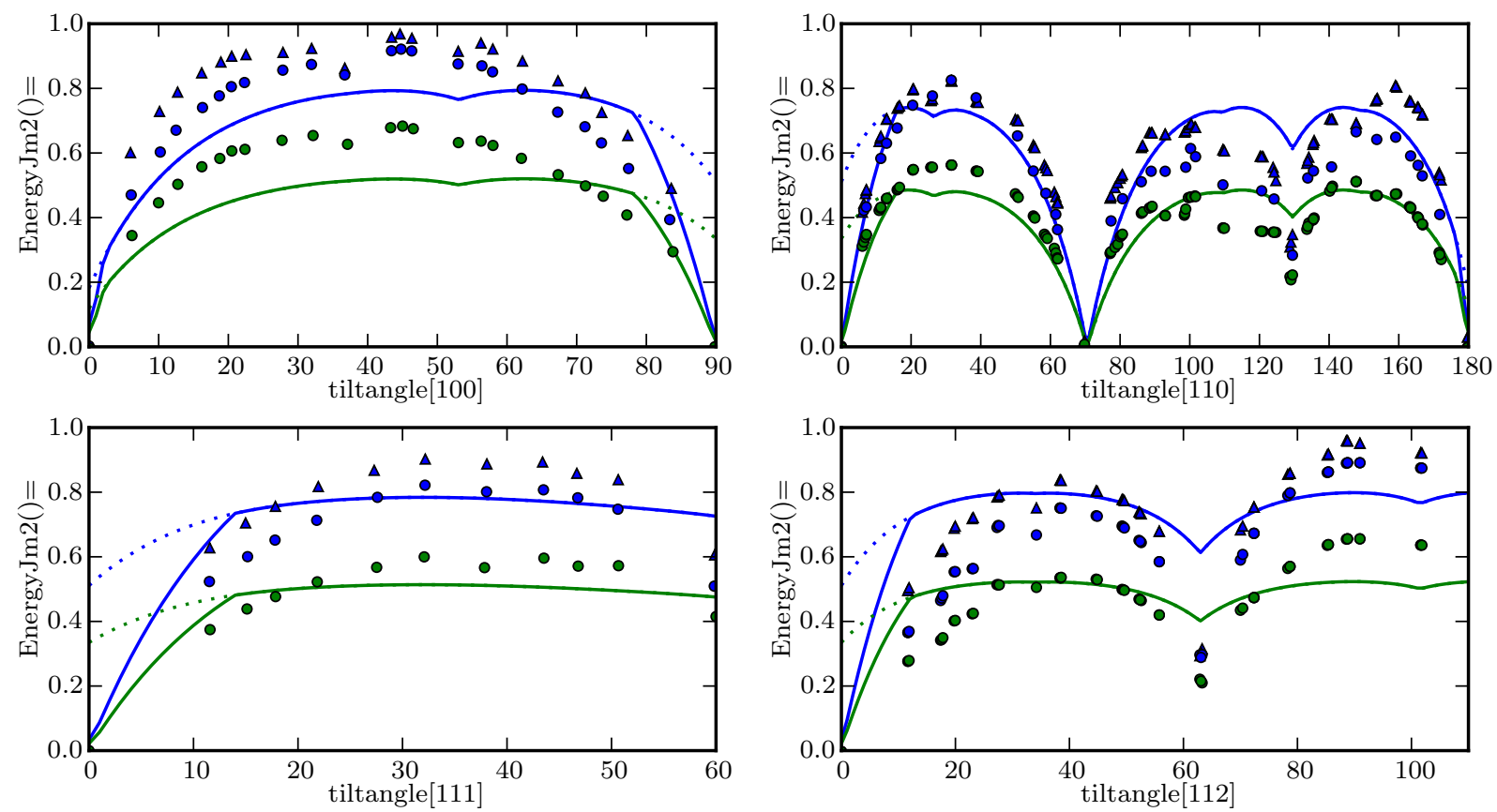

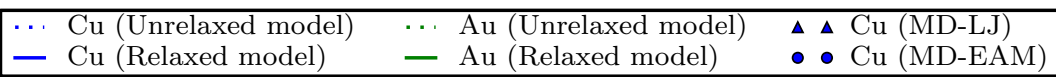

Figure 4: FCC STGB results of the covariance model (relaxed and unrelaxed) for copper and gold, compared with MD results using the Embedded Atom Method (EAM) and Lennard-Jones (LJ) potentials. MD results from [45]. Annotations on the top indicate the boundary plane for the top crystal at cusp locations.

(blue) and $\alpha$ phase Iron (green). As with the FCC results, the results from the unrelaxed model are plotted with a hashed line, while the relaxed model results are plotted using a solid line. For almost the entire range of tilt angles for all four cases, no relaxation occurs and the relaxed plot is consequently coincident with the unrelaxed plot. The only relaxation that is observed to occur in these figures is: for [100] tilt, $0^{\circ} \leq \theta<5^{\circ}$; for [110] tilt, $175^{\circ}<\theta \leq 180^{\circ}$. The reason for the relaxation occurring exclusively for these configurations is intuitive and is consistent with that of the FCC tilt case. Evaluation of the covariance model in the uniform crystal case shows that there exists an anisotropy in the energy. The absolute minimum energy value is attained in the uniform crystal orientation corresponding to the most densly packed plane: for FCC the $\{111\}$ plane and for BCC the $\{110\}$, and the energy for all uniform crystal orientations is reduced to zero by convexifying between these minimal energy orientations. As a result, no relaxation occurs in the BCC STGB case when the uniform crystal boundary is already minimized; because $\theta=0$ corresponds to a $\{110\}$ plane for most of the cases, no relaxation occurs.

At this point the reader may raise an objection regarding the consistency of the use of a relaxation construction with symmetric tilt grain boundaries: STGBs are generally observed to be planar, any faceting construction of the interface results in a departure from observed (and simulated) behavior. In response we remark that all relaxation for STGBs occurs at or very near to the uniform crystal case. Uniform crystals by definition have no physical boundary; the supposed interface between them is reduced to a construction. Thus there is no reason why the constructed interface need be planar; on the contrary, because we expect the constructed boundary in a uniform crystal to be densely packed, we would expect that it follows the densely packed regions to some extent. 

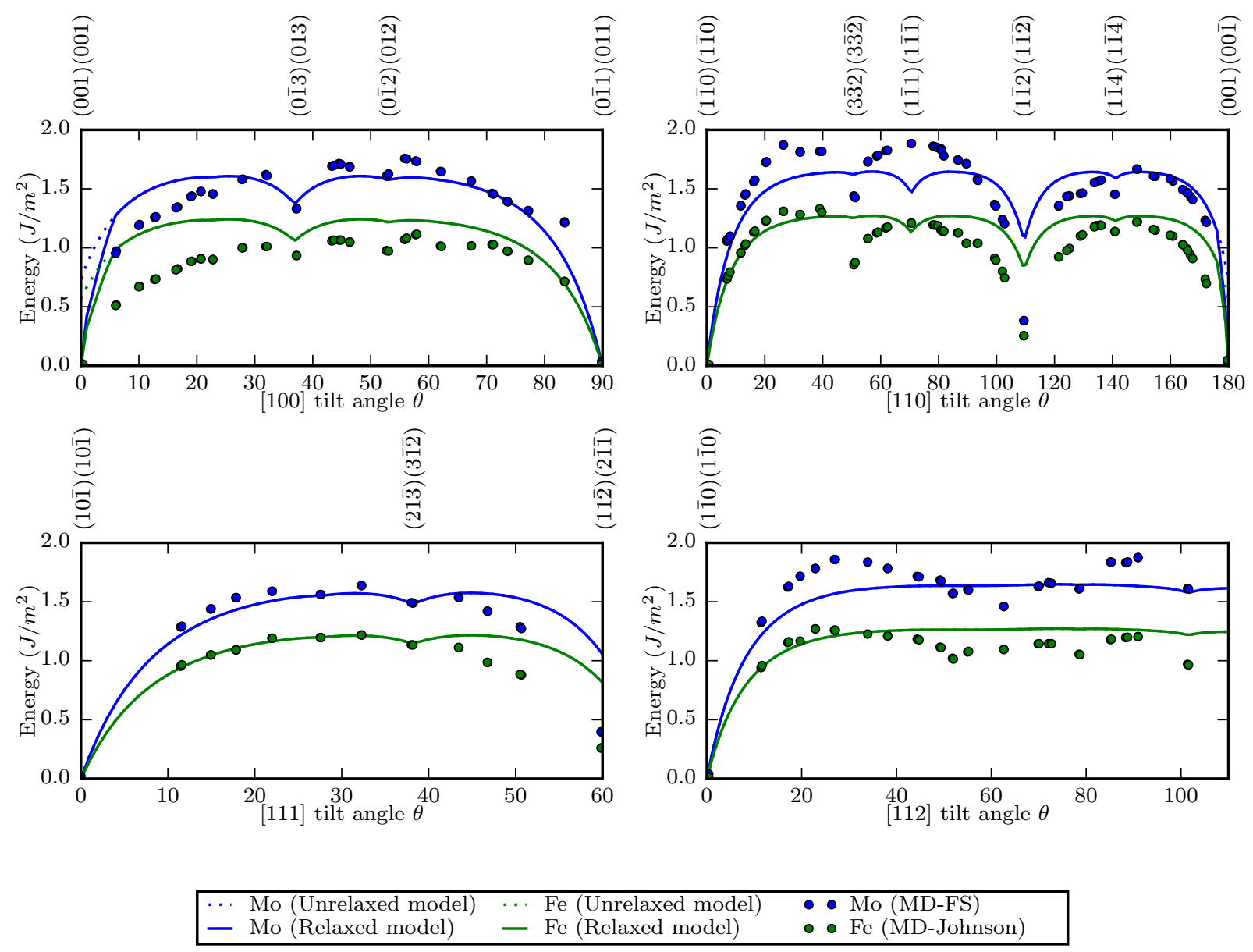

Figure 5: BCC STGB results of the covariance model (relaxed and unrelaxed) for molybdenum and $\alpha$ Iron, compared with MD results using FS (for Mo) and Johnson (for Fe) potentials. MD results from [46]. Annotations on the top indicate the boundary plane for the top crystal at cusp locations.

We also note that relaxation does not occur for the majority of the tilt configurations. That is, the energy surface for most STGBs is sufficiently convex so that it is not possible to reduce the energy by faceting. Consequently we conclude that the observed planar behavior of STGBs may be explained by the conclusion that unrelaxed STGBs (except in the uniform crystal case) are energy minimizers.

The relaxation effectively resolves a significant discrepancy in the original covariance model, the inability to give the correct value of zero energy for all uniform crystal boundaries. The effect of this differs between cases. For all of the symmetric tilt grain boundaries, and for the FCC cases in particular, we observe that the covariance model used with the relaxation theory produces results that very closely match results from MD calculations.

\subsection{Twist grain boundaries}

For every possible grain boundary there exists as set of twist grain boundaries (TwGB) corresponding to it. TwGBs are generated by rotating the top and bottom crystals of any grain boundary antisymmetrically about a twist axis that is normal to the interface plane (Figure 6.) Any TwGB is described by its two boundary planes and the twist angle. A subset of the family of TwGBs are symmetric twist grain boundaries (STwGB). A TwGB is a STwGB if the crystal is uniform when the twist angle is $\theta=0^{\circ}$. A TwGB is specified by a single boundary plane. Finally, the set of asymmetric twist grain boundaries (ATwGB) are all the TwGBs that are not STwGBs. 


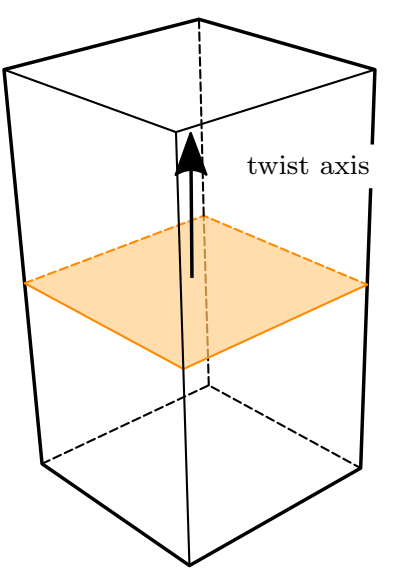

(a) Begin with a uniform crystal

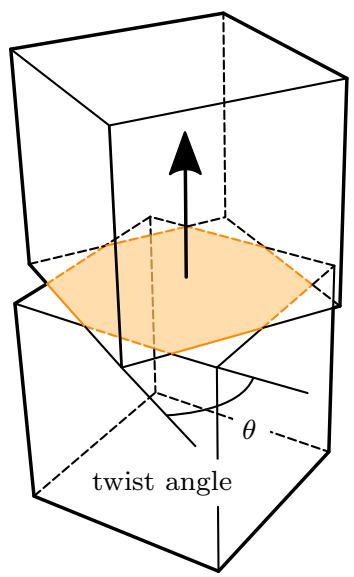

(b) Rotate symmetrically about tilt axis to form STGB

Figure 6: Geometry of asymmetric tilt grain boundaries

The selection of TwGBs that we use for comparison is driven by the availability of MD data. For FCCFCC TwGBs we compare with two STwGB cases and four ATwGB cases. The results for the comparison are included in Figure 7. As with the previous section, the unrelaxed results are represented with a hashed line, and the relaxed with a solid line. Only data for copper was available for the FCC STwGB case, so we only include the results for copper STwGB. For the remainder of the cases we compare results for both copper and gold. As with the previous work, we observe the greatest discrepancy in the case of the FCC STwGBs. While the shape and cusp location match well, both appear to differ by some multiplicative constant.

For the (111) case, no relaxation occurs because, as expected, the unrelaxed model attains its minimum for $\theta=0^{\circ}$. For the (100) case we observe relaxation occuring near $\theta=0^{\circ}, 90^{\circ}$. As with STGBs, relaxation using $\{111\}$ facets in FCC materials is necessary to correctly recover the value of 0 for uniform crystals. For the (115)(111) and (221)(001) asymmetric twist we observe very good agreement between the MD data and the results of the model. The improvement on the (115)(111) case by relaxation is negligible but unnecessary given the high correlation of the unrelaxed results. For (221)(001), the unrelaxed model overpredicts the value of the energy at the cusps for $\theta=0^{\circ}, 90^{\circ}$; however the relaxed model corrects the energy value to give an almost exact matching of the cusp magnitude. For the remaining two ATwGBs we observe more chaotic energy data in the MD results as well as the relaxed and unrelaxed model. For the (114)(011) ATwGB we see a marked difference between the behavior of the MD data for copper vs for gold, indicating that for this type of interface geometry may not be the main factor influencing GB energy. We see a considerable improvement on the unrelaxed model at the energy cusps for $\theta=0^{\circ}, 180^{\circ}$. The model already predicted cusps at those locations, but relaxation corrects the magnitudes for an almost exact match. For (557)(113), there is a slight improvement in the energy for the cusps at $\theta=0^{\circ}, 180^{\circ}$, though the energy match is not exact. It is interesting to note that small energy reductions by relaxation occur through the range of $\theta$ for both (114)(011) and 557)(113) TwGBs. An examination of the P3 visualizations for those values do not yield any interesting insight into their morphology, but we observe that continuous, slight relaxation seems to correspond to more chaotic boundaries that invariable have more chaotic morphology. This is a trend that we will observe in the following BCC twist cases as well.

A broader range of BCC twist grain boundary data is available, giving us a greater dataset for validation. For symmetric twist only data for alpha-phase iron was found, so we include only the iron results. For asymmetric twist we compare against both alpha-phase iron and molybdenum.

The results for BCC symmetric twist grain boundaries are plotted in Figure 8. As we expect for (100) symmetric twist relaxation occurs near the end cusps, as a result of the fact that (100) boundaries for 

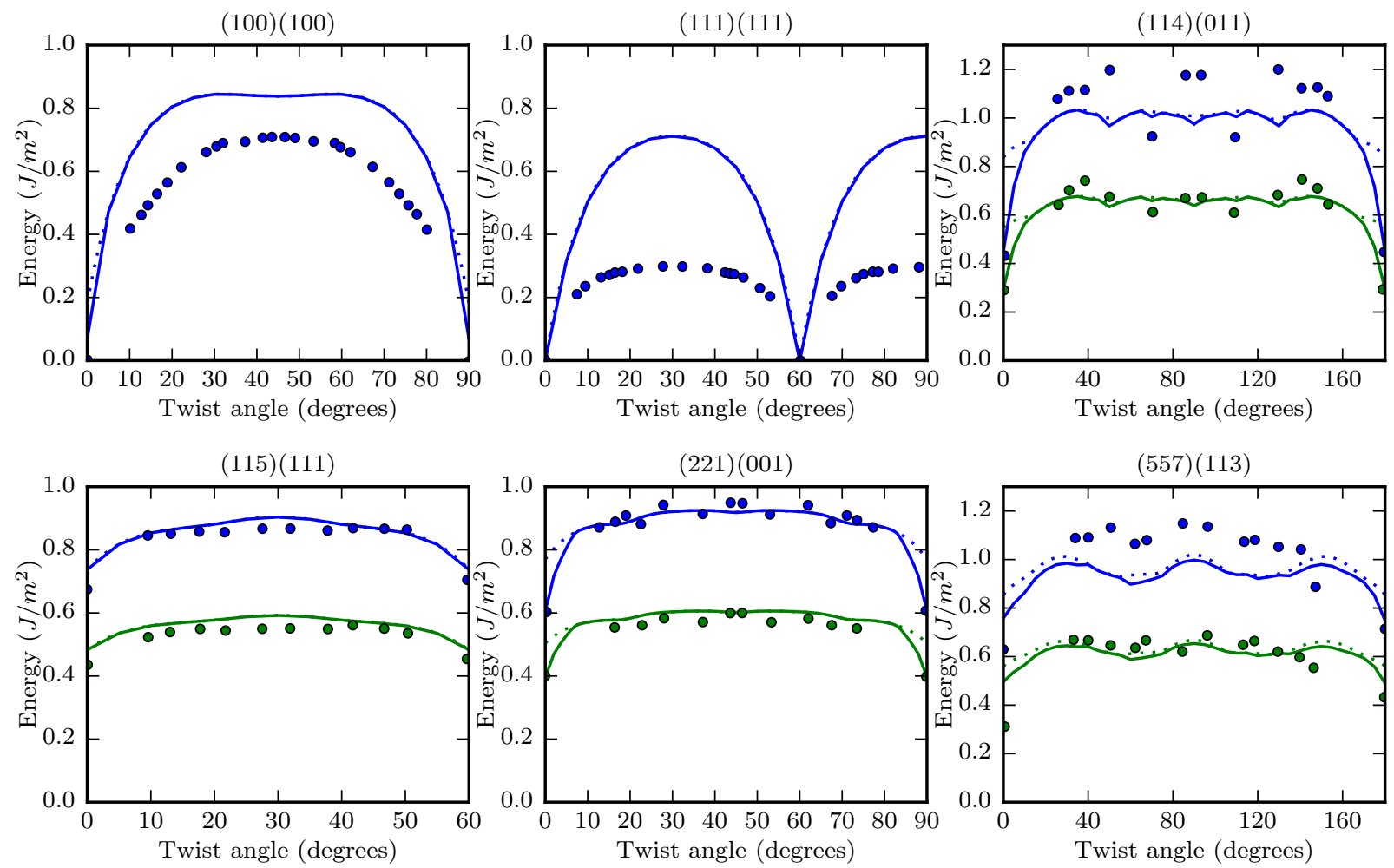

\begin{tabular}{|lll|}
\hline$\cdots \mathrm{Cu}(\mathrm{Cov})$ & $\cdots \mathrm{Au}(\mathrm{Cov})$ & $\bullet \bullet \mathrm{Cu}(\mathrm{MD}-\mathrm{LJ})$ \\
$-\mathrm{Cu}(\mathrm{RCov})$ & $-\mathrm{Au}(\mathrm{RCov})$ & $\bullet$ \\
\hline
\end{tabular}

Figure 7: FCC TwGB results of the covariance model (relaxed and unrelaxed) for copper and gold, compared with MD results using EAM (for gold) and LJ (for copper). The boundary planes (constant for all twist angles) are indicated by the graph labels. MD results for symmetric twist from [47], asymmetric twist from [48].

uniform crystals in BCC require convexification with (110) boundaries to reduce the energy to zero. For the remainder of this region the match is close, and coincident with the unrelaxed model. No relaxation occurs for the (110) STwGB set of boundaries; the unrelaxed model recovers $\gamma=0$ at the end cusps and apparently the remainder of the interfaces are planar. As in the previous work we note that the shape of the energy matches very closely, differing only by a multiplicative constant. For the (031)(031) symmetric twist, the relaxation model correctly adjusts the cusp at $\theta=0^{\circ}$ to zero. The resultant matching of both end cusps is very good. We see a fairly chaotic pattern in both the MD and the model data, with the relaxed energy diverging slightly from the unrelaxed at multiple points. The match of the general trend is good, and neither MD nor model predict the existence of a well-defined cusp that the other does not. For the (111)(111) symmetric twist we find again that the model correctly adjusts the $\theta=0^{\circ}$ to zero as expected; no other relaxation occurs, and the matching of the energy trends and cusp magnitudes is very close. Finally, as with most of the other cases, we observe $\theta=0^{\circ}$ cusp correction to zero for (112)(112).

The results for BCC asymmetric twist grain boundaries are plotted in Figure 9. For the (114)(011) twist almost an exact match exists between the unrelaxed model and the MD data. No relaxation is observed, (or needed). For the (115)(111) twist we observe a slight reduction of energy by relaxation for $\theta<50^{\circ}$, and a significant reduction at the cusp $\theta=0^{\circ}$. The cusp matching is not precise, but the relaxation clearly improves the unrelaxed results considerably. For (158)(031), we observe a significantly chaotic pattern in both MD data and the model. The relaxation behavior is unique for this set of data in that additional 
(001)(001)

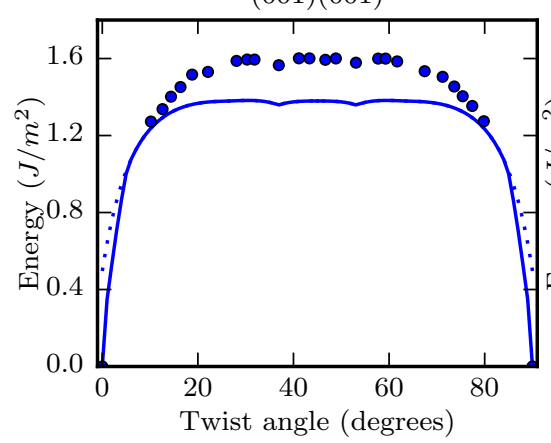

(111)(111)

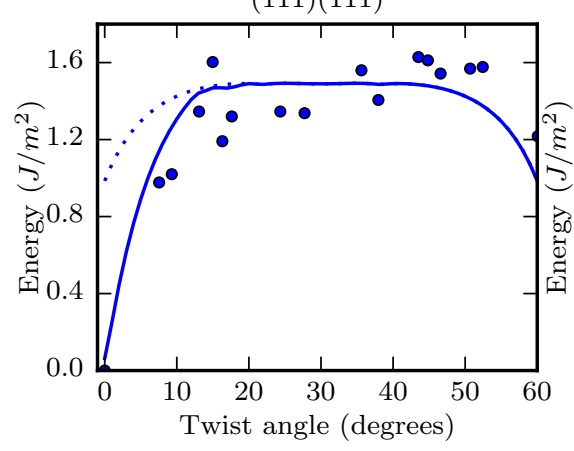

(011)(011)

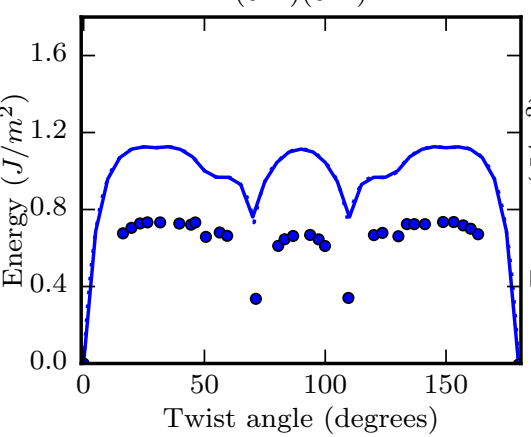

(112)(112)

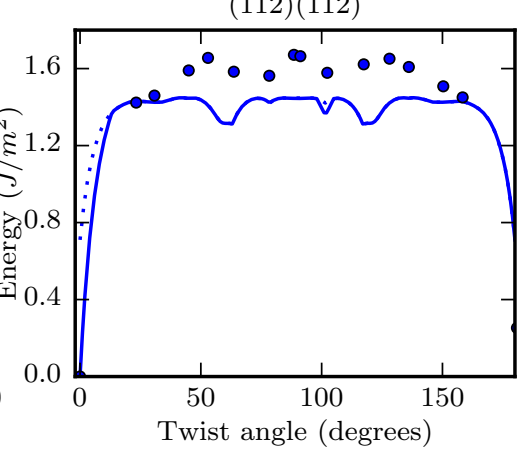

(031)(031)

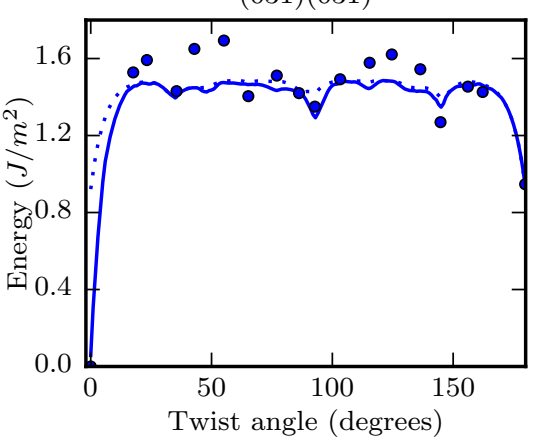

Figure 8: BCC StwGB results of the covariance model (relaxed and unrelaxed) for $\alpha$-Iron, compared with MD results using a Johnson potential. MD data from $[49,50]$

minima are formed by the creation of relaxation structures. Additionally we do not observe any correction of the values at the end points, although neither endpoint appears to correspond strongly to a cups in either the model or MD results. For the (221)(001) case we observe no relaxation and, interestingly, the value of the energy at the cusps does not change either. Good agreement is observed for molybdnum in particular. An energy structure in the MD data is observed for iron that differs from molybdnum. This is very similar to the pattern in copper and the difference between it and gold in (114)(001) asymmetric twist, shown previously. As with the FCC case, this difference may indicate a departure from the interface energy behavior from dependence on geometry, at least for this type of grain boundary. Finally, for the (552)(112) case we observe majority coincidence of the unrelaxed model with the relaxed model. However, relaxation occurs near $\theta=180^{\circ}$ that improves the matching of the energy cusps. Otherwise we find good agreement between the model and the MD results.

In conclusion for the discussion of twist grain boundaries, we believe that the combination of the covariance model with relaxation produces a wide range of results that match very well with MD data. Some outlying cases exist. However, we find almost universal agreement of the cusp location. Because the model was designed primarily as a means of locating cusps, this serves as validation within the mission space of the model. Further, the primary discrepancies between the model and the MD data are generally cases where the results are still almost identical up to a multiplicative constant. Closer matching could be obtained by adjusting the model parameters for those sets specifically, but a departure from a consistent set of parameters would compromise the model's predictive ability. A number of explanations may be offered for these differences ranging from the inability of the model to access certain relaxation mechanisms, to an inaccuracy of the MD potentials [50]. 
$(114)(011)$

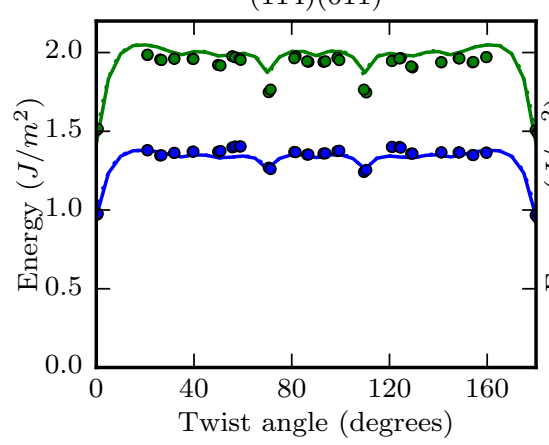

$(221)(001)$

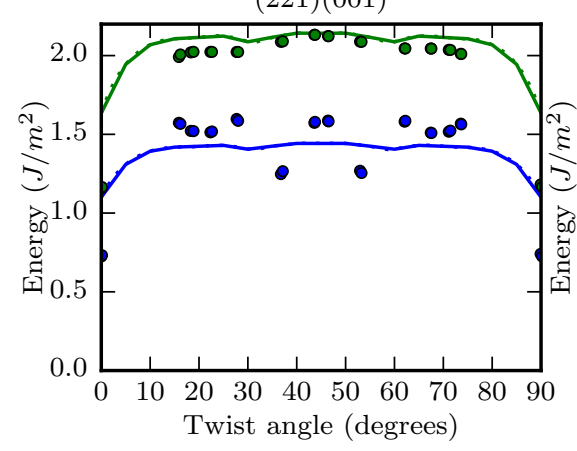

(115)(111)

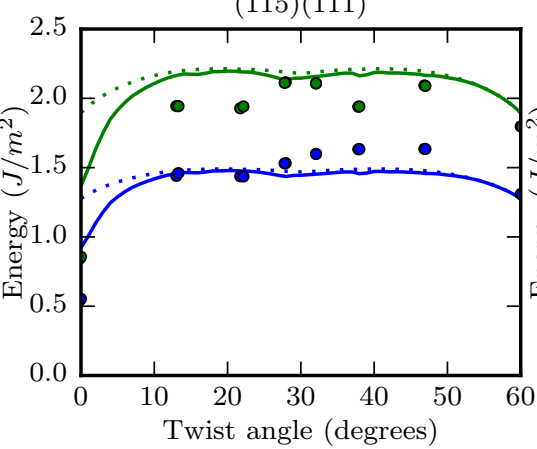

$(552)(112)$

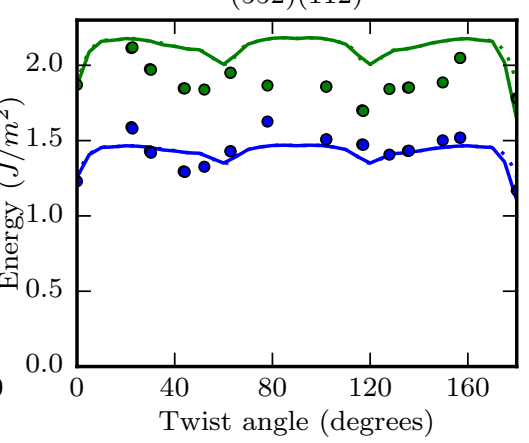

$(158)(031)$

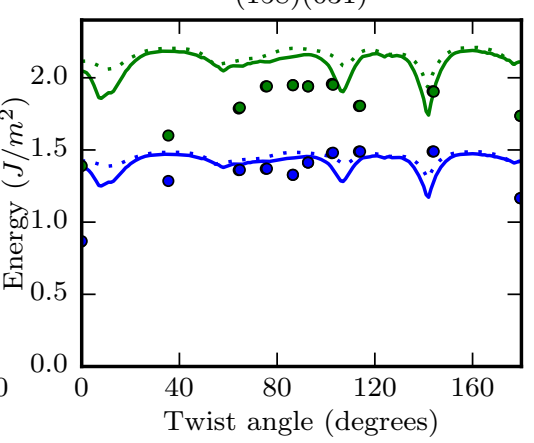

‥ Mo (Unrelaxed model)

Fe (Unrelaxed model)

-

- Mo (MD-FS)

- Fe (MD-Johnson)

Figure 9: BCC ATwGB results of the covariance model (relaxed and unrelaxed) for molybdenum and $\alpha$ Irdon, compared with MD results using a Finnis-Sinclair (FS) potential for Mo and a Johnson potential for FE. MD results from $[50]$

\subsection{Asymmetric tilt grain boundaries}

The last set of grain boundaries that we consider are the set of asymmetric tilt grain boundaries (ATGBs). The space of possible ATGBs is enormous, encompassing almost any type of grain boundary. In this work we restrict our use of the ATGB nomenclature to a particular type of asymmetric tilt grain boundary.

ATGBs are defined by the way in which they are constructed. Figure 10 illustrates the procedure for generating an ATGB from a uniform crystal. The uniform crystal boundary is transformed to a symmetric tilt grain boundary by a symmetric rotation about an in-plane axis. Then a different in-plane axis is selected and the interface is rotated with the orientation relationship of the crystals remaining fixed. (Alternatively, the top and bottom crystals are tilted antisymmetrically about the in-plane axis.) The axis about which the interface is rotated is referred to as the inclination axis, and the angle of rotation $\phi$ as the inclination angle. In this work we refer to a class of ATGBs using the following system: a $\{l m n\}\langle p q r\rangle$ set of ATGBs is one for which $\phi$ corresponds to inclination of the interface about a mutual $\langle p q r\rangle$ axis, and $\phi=0^{\circ}$ corresponds to a $\{l m n\}$ STGB. One major disadvantage to this categorization system is that ATGBs can sometimes be constructed more than one way, resulting in more than one classification.

ATGBs are of interest because they are relatively simple and have a high degree of symmetry, yet tend to have complex morphology. ATGBs are ideal for observing the behavior of the relaxation model to determine its ability to predict not only the relaxed energy but the corresponding morphology as well. In this work we study three different ATGBs. As before, our choice of GB cases is driven by the availiability of published experimental and MD data. For the first two cases, $\{111\}\langle 110\rangle$ and $\{111\}\langle 112\rangle$, we compare energetic data with results from MD and compare the morphological prediction of the relaxed model with experimental observation. For the final case, $\{113\}\langle 110\rangle$, we are not aware of energetic data for comparison. For this case instead, we examine the effect of changing the dimensionless temperature $\sigma$ to determine its effect on 


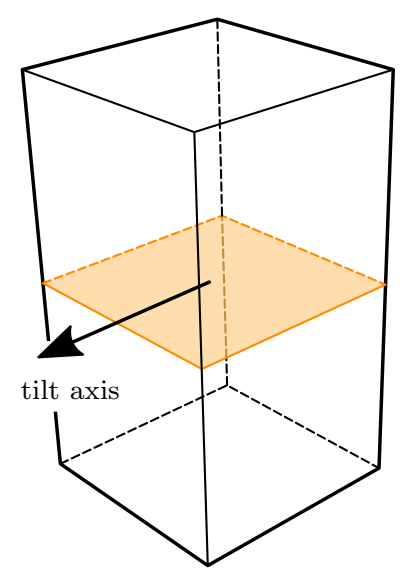

(a) Begin with a uniform crystal

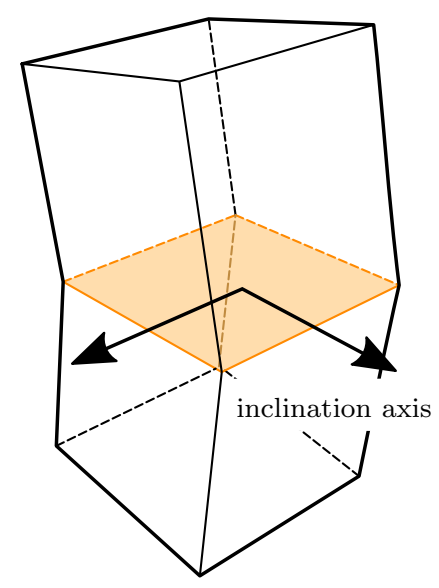

(b) Rotate symmetrically about tilt axis to form STGB

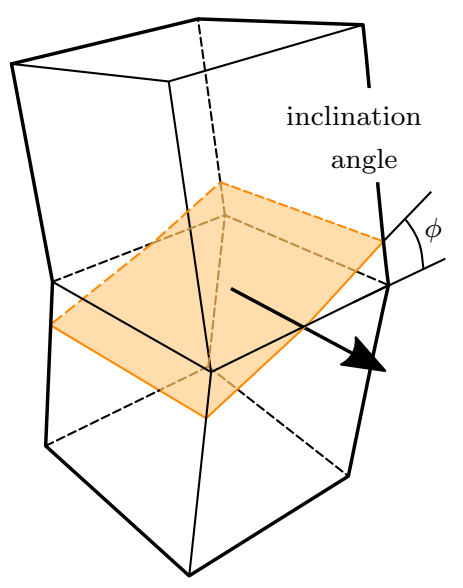

(c) Rotate interface about inclination axis to form ATGB

Figure 10: Geometry of asymmetric tilt grain boundaries

faceting behavior and the relationship to thermalization in experimental observation.

\subsection{1. $\{111\}\langle 110\rangle A T G B$}

The energetic results for the $\{111\}\langle 110\rangle$ ATGB for various FCC materials are shown in Figure 11. Comparison is made of copper and aluminum data with MD results, and the relaxed results for gold are included as well. As with the other tests the unrelaxed results are plotted as a hashed line with the relaxed results plotted with a solid line. (Only the unrelaxed results for copper are included; including it for gold and aluminum occludes the other plots.) We observe a major difference between the unrelaxed and the relaxed results. Order 2 faceting occurs for all points $0^{\circ}<\phi<90^{\circ}$, where facets are created corresponding to the $0^{\circ}$ and $90^{\circ}$ orientations. The energy appears to match closely, and the resultant 2 -facet pattern is consistent with observation of the MD results.

In the original paper containing the MD data, Wolf proposed a scheme for approximating the energy of the faceted interface given the energy per unit area of the two individual facets [28]. Here we note that this formula is recoverable as a special case of the relaxation model. Let $\gamma(\phi)$ be the unrelaxed energy as a function of inclination angle, and let $\phi_{1}=0^{\circ}$ and $\phi_{2}=90^{\circ}$ correspond to the optimal facet orientations. Then the optimal normal vectors are

$$
\boldsymbol{n}_{1}(\phi)=\left[\begin{array}{c}
0 \\
\sin \left(\phi_{1}-\phi\right) \\
\cos \left(\phi_{1}-\phi\right)
\end{array}\right]=\left[\begin{array}{c}
0 \\
-\sin (\phi) \\
\cos (\phi)
\end{array}\right], \quad \quad \boldsymbol{n}_{2}(\phi)=\left[\begin{array}{c}
0 \\
\sin \left(\phi_{2}-\phi\right) \\
\cos \left(\phi_{2}-\phi\right)
\end{array}\right]=\left[\begin{array}{c}
0 \\
\cos (\phi) \\
\sin (\phi)
\end{array}\right]
$$

We use the constraint of the optimization problem to obtain $\lambda_{1}, \lambda_{1}$ :

$$
\left[\begin{array}{cc}
-\sin (\phi) & \cos (\phi) \\
\cos (\phi) & \sin (\phi)
\end{array}\right]\left[\begin{array}{l}
\lambda_{1} \\
\lambda_{2}
\end{array}\right]=\left[\begin{array}{l}
0 \\
1
\end{array}\right] \Longrightarrow\left[\begin{array}{l}
\lambda_{1} \\
\lambda_{2}
\end{array}\right]=\left[\begin{array}{l}
\cos (\phi) \\
\sin (\phi)
\end{array}\right]
$$

Then the relaxed energy is

$$
\gamma(\phi)=\cos (\phi) \gamma\left(\phi_{1}\right)+\sin (\phi) \gamma\left(\phi_{2}\right),
$$

which corresponds to Wolf's approximation where $\gamma_{1}=\gamma\left(\phi_{1}\right), \gamma_{2}=\gamma\left(\phi_{2}\right)$. 


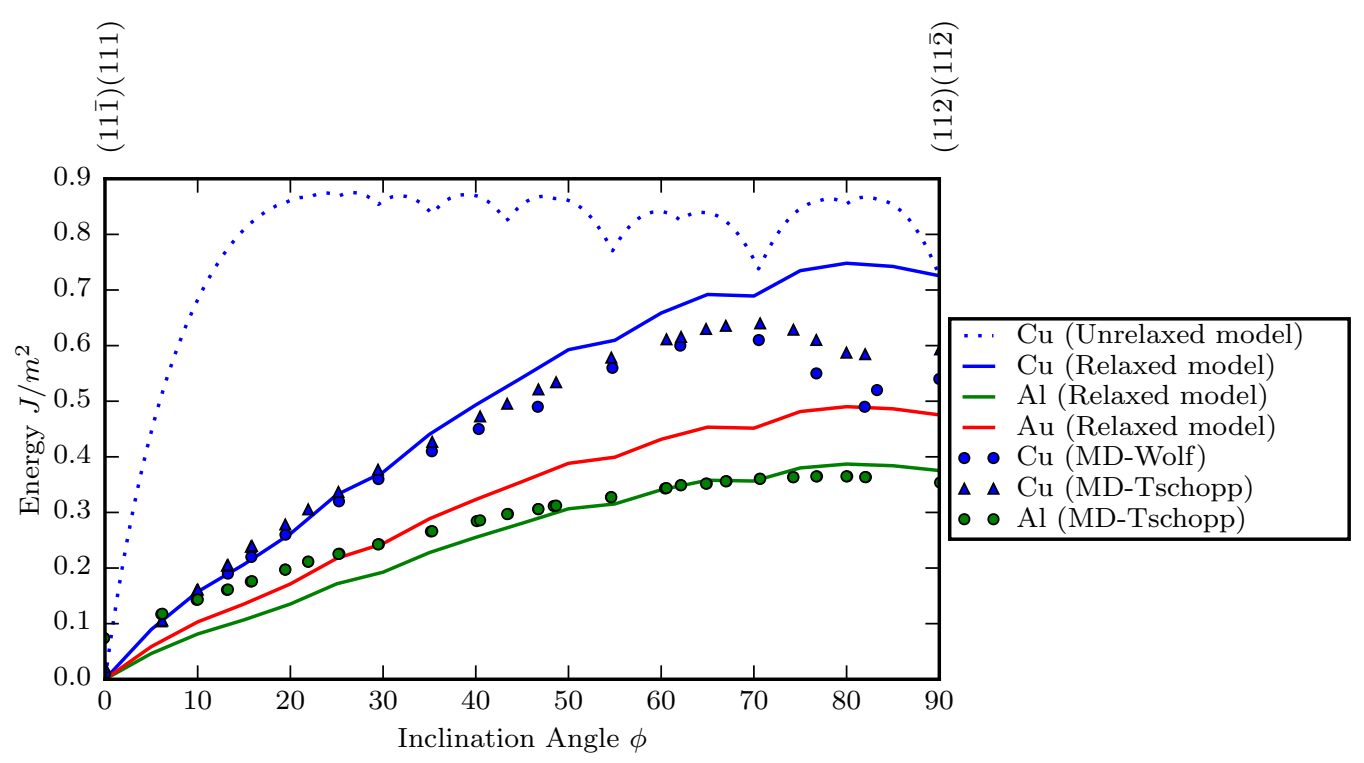

Figure 11: MD data for $\mathrm{Cu}$ from [28, 51]. MD data for $\mathrm{Al}$ from [51]

\subsection{2. $\{111\}\langle 112\rangle A T G B$}

Next we consider a similar set of ATGB data. These grain boundaries are obtained by beginning with the same STGB as in the previous section, but inclining the interface about the mutual $\langle 112\rangle$ axis instead of the $\langle 110\rangle$ axis. This family of ATGBs demonstrates a wide range of morphological behavior, varying from planar in the STGB case to 3-faceted for $0^{\circ}<\phi<90^{\circ}$ to 2 -faceted for $90^{\circ}$. The evolution of the F3 plot and the corresponding morphology is given in Figure 12.

One particularly interesting thing to note about the evolution of this morphology is that the 2-facet direction for $\phi=90^{\circ}$ is orthogonal to the inclination axis. As a result, an observer looking at a 2D cross section with the inclination axis as a normal will not be able to detect the 2-facets. However, the case of $\phi=90^{\circ}$ is interesting because it lies in the intersection of $\{111\}\langle 112\rangle$ ATGBs and $\{112\}\langle 111\rangle$ ATGBs.

$\{112\}\langle 111\rangle$ ATGBs have been studied experimentally. The relaxation model gives the precise orientation of the facets formed for the $90^{\circ}$ case, and can be used to construct a faceting pattern. In Figure 13, experimental observations of faceting in this interface configuration are compared with results from the model by overlaying the observed interface with a reconstructed one. We observe that the matching is almost exact.

Finally, we compare the computed relaxed energy of the ATGB with that determined using MD in Figure 14. We observe that the energy match is consistent, although the MD data increases sharply as $\phi \rightarrow 90^{\circ}$. Because the MD results for $\phi=90^{\circ}$ did not indicate the presence of 2 -faceting, one possible reason for the difference may be that the MD results were high because the system did not relax. Nevertheless, we conclude that the experimental faceting matches well with the predicted faceting and that the MD data matches well with the predicted relaxed energy.

\subsection{3. $\{113\}\langle 110\rangle A T G B$}

Here we investigate the effect of thermalization on faceting configurations. We compare with results from Hsieh and Balluffi who examined the results of temperature on faceted interfaces, and concluded that a de-faceting transition can take place as the result of elevated temperature [52]. The thermal regularization in the covariance model admits a convenient way of examining the effect of elevated temperature on grain boundaries. If a quadratic potential is used for $w$ (the energy potential used for the thermalization mollifier) then the parameter $\sigma=k_{B} T / w_{0}$ models the dimensionless temperature of the interface. We consider the effect of thermalization on the $\{113\}\langle 110\rangle$ asymmetric tilt grain boundary in $\mathrm{Al}$, where the inclination angel 


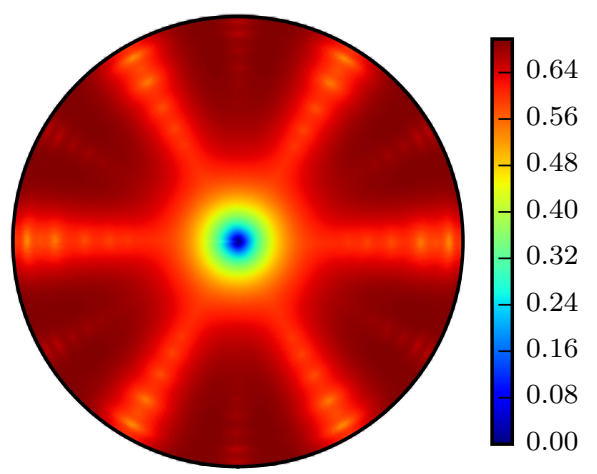

(a) Projected polar plot of energy for $\phi=0^{\circ}$

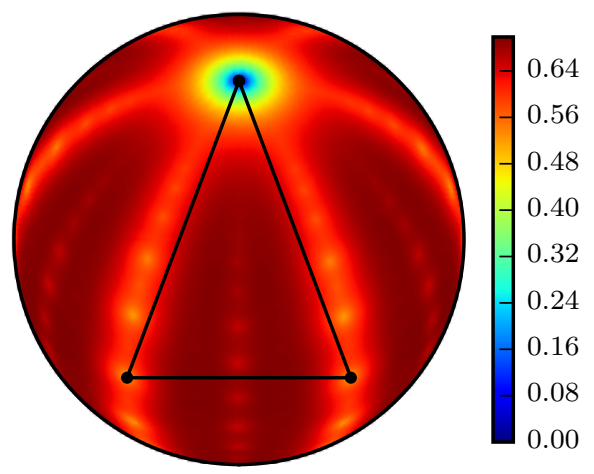

(c) Projected polar plot of energy for $\phi=45^{\circ}$

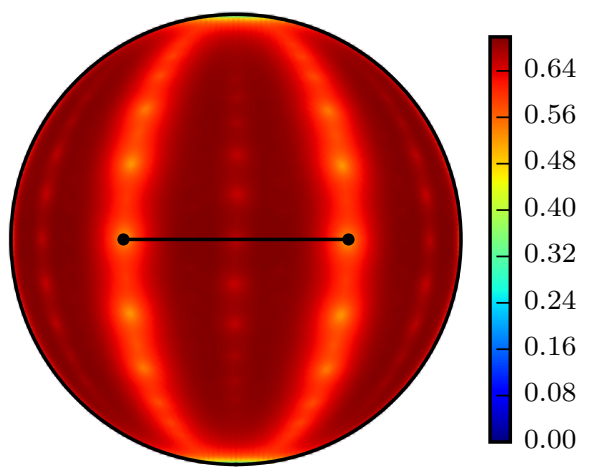

(e) Projected polar plot of energy for $\phi=90^{\circ}$

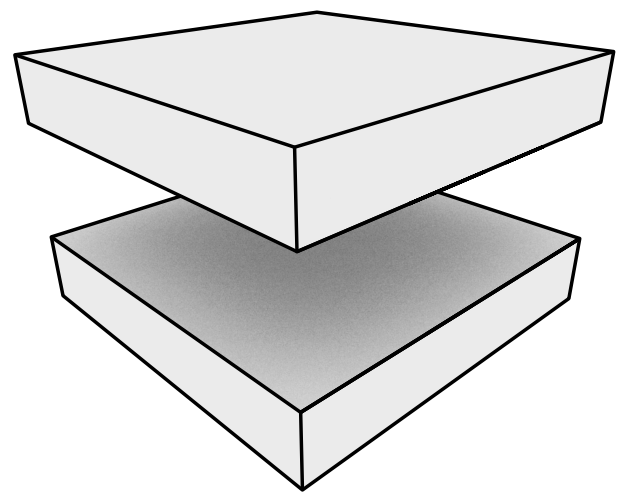

(b) Facet pattern for $\phi=0^{\circ}$

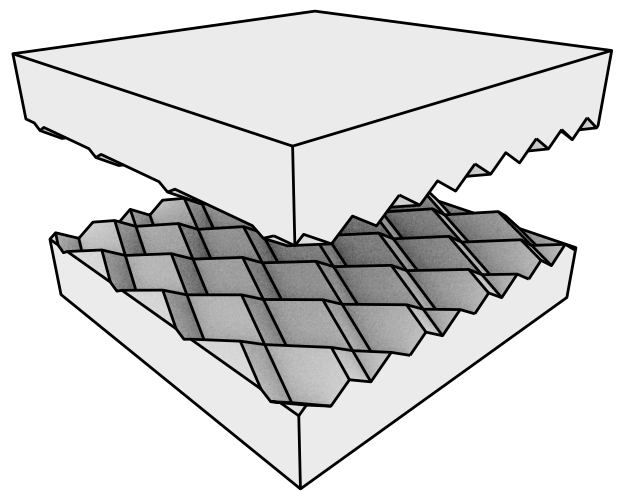

(d) Facet pattern for $\phi=45^{\circ}$

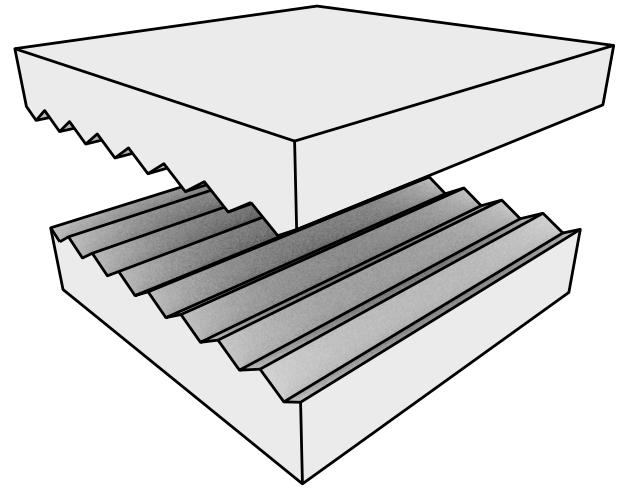

(f) Facet pattern for $\phi=90^{\circ}$

Figure 12: Evolution of the facet microstructure for $\phi$ varying from $0^{\circ}$ to $90^{\circ}$. 
is $25.24^{\circ}$ so that the mean ("prescribed") upper and lower boundary planes are $\{002\}$ and $\{667\}$.

For interfaces of this type we find that there are no facet patterns of order greater than two. Thus we restrict to two-dimensional faceting, which speeds up the analysis. In Figure 15 we plot the energy for several values of $\sigma$ and all rotations of the interface about the shared [110] axis. The thin blue curve corresponds to the unrelaxed energy for the ATGB as a function of interface orientation, where the value at $\theta=0^{\circ}$ (the "north" end of the plot) is the energy for the planar interface without relaxation.

The thick blue line corresponds to the relaxed energy for each rotation of the interface. As expected, we recover the maximal convex energy (in the sense of crystalline integrands [14]), i. e., the convexification $\gamma_{C}$. In general we find that computation of $\gamma_{C}$ is infeasible for the full range of $\mathbf{n} \in \mathcal{S}_{2}$; however it is possible here by restriction to $\mathbf{n} \in \mathcal{S}_{1} \subset \mathcal{S}_{2}$.

Also of interest (albeit extraneous to the observation of faceting structure) is the crystal of the energy, or the Wulff shape, shown as the shaded region. The Wulff shape is a construction corresponding to the energy-minimizing shape of an inclusion given a specified volume; that is, the optimal shape that one crystal would take if it is an inclusion in the other. Here the boundary of the Wulff shape is computed by solving

$$
\gamma_{c}(\boldsymbol{n})=\inf _{\overline{\boldsymbol{n}} \in S_{1}} \frac{w(\overline{\boldsymbol{n}})}{\overline{\boldsymbol{n}} \cdot \boldsymbol{n}} .
$$

Clearly the Wulff shape undergoes significant changes as the temperature increases. We see that for higher temperatures the structure becomes more amorphous, whereas lower temperatures produce a crystal with sharper edges.

We observe that as the temperature increases the minimizing facet pattern changes. For $\sigma=0.1,0.15$ the optimal facets form at $\theta=4.26^{\circ},-25.24^{\circ}$ corresponding to interfaces with $(001)(6 \overline{6} 7)$ and $(1 \overline{1} 3)(\overline{1} 13)$ boundary planes, respectively. In Figure 16a we observe that this faceting construction corresponds very well to the observed morphology. On the other hand, for $\sigma=0.2,0.25$ the $\{113\}$ STGB boundary plane facets remain, but the other facet plane is the incoherent $\sim(1 \overline{1} 19) \sim(37 \overline{3750})$ boundary. In Figure 16b we observe that the high temperature faceting pattern is also observed experimentally.

We note the following observations. 1) The faceting transition behavior occurs independently of the value of $E_{0}$, which acts as a scaling constant and does not affect the convexification. 2) Material dependence can be introduced via the $w_{0}$ parameter, the atomic embedding energy constant. As a result we expect that a faceting phase transition of this type will take place in all FCC materials, but the transition temperature for different materials will differ for different values of $w_{0}$.

\section{Summary and concluding remarks}

The purpose of the work presented in the foregoing is twofold: To review and reformulate the theory of interfacial energy relaxation with a view toward predicting relaxed energies and morphologies of crystalline interfaces, and to validate the relaxation approach, in conjunction with the covariance model of interfacial energy, through comparisons with a wide range of existing experimental and MD data. It bears emphasis that, in conducting those comparisons, the same set of three parameters has been used for all cases involving the same material: they are not adjusted on a case-by-case basis. We have specifically applied the relaxation model to FCC/BCC, symmetric/asymmetric, tilt/twist boundaries, and obtained a match with energy and morphology data in all cases.

We find the ability of a simple model of interfacial energy and a general microstructure construction based on convexification to replicate complex interfacial morphologies, including thermally-induced morphological transitions, to be gratifying. Given the strength of the validation results, it seems appropriate to conclude that the energy of crystal interfaces is geometrically determined. In particular, it appears that a simple two-point geometrical measure, the covariance of the atomic densities, correlates with the interfacial energy. Furthermore, the close agreement between the predictions of the faceting construction and experimental observation strongly suggests that the morphology of crystal interfaces is driven by energy minimization.

We also note that the present form of the model is essentially analytical and, therefore, exceedingly fast to evaluate. This computational efficiency opens the way for the implementation of the model in continuum- 
level codes to study interface evolution on a large scale, and in multiscale models of crystal plasticity and martensite where interfacial energy plays a critical role in determining microstructural size and morphology.

\section{Acknowledgments}

Brandon Runnels and Michael Ortiz would like to thank the NNSA's High Energy Density Laboratory Plasmas program under award \#DE-NA0001805. Brandon Runnels additionally thanks the Los Alamos National Laboratory Seaborg Institute for support during Summer 2014. Irene Beyerlein would like to acknowledge support by a Laboratory Directed Research and Development program award number 20140348ER. Sergio Conti would like to acknowledge support of the DFG unter SFB 1060, project A6.

\section{References}

[1] I. J. Beyerlein, J. Wang, K. Kang, S. J. Zheng, and N. A. Mara, "Twinnability of bimetal interfaces in nanostructured composites," Materials Research Letters, vol. 1, pp. 89-95, June 2013.

[2] S. Zheng, J. S. Carpenter, J. Wang, N. A. Mara, and I. J. Beyerlein, "An interface facet driven rayleigh instability in high-aspect-ratio bimetallic nanolayered composites," Applied Physics Letters, vol. 105, no. 11, p. 111901, 2014.

[3] M. J. Demkowicz and L. Thilly, "Structure, shear resistance and interaction with point defects of interfaces in CuNb nanocomposites synthesized by severe plastic deformation," Acta Materialia, vol. 59, pp. 7744-7756, Dec. 2011.

[4] D. A. Molodov, T. Gorkaya, and G. Gottstein, "Dynamics of grain boundaries under applied mechanical stress," Journal of Materials Science, vol. 46, pp. 4318-4326, Jan. 2011.

[5] D. A. Molodov, T. Gorkaya, and G. Gottstein, "Migration of the $\Sigma 7$ tilt grain boundary in Al under an applied external stress," Scripta Materialia, vol. 65, pp. 990-993, Dec. 2011.

[6] Z. Hong-da, W. Xiao-dan, W. Chong-ming, J. Bo, and X. Hai-long, "Selective SVM Ensembles Based on Modified BPSO," in 2008 IEEE Pacific-Asia Workshop on Computational Intelligence and Industrial Application, vol. 1, pp. 243-246, IEEE, Dec. 2008.

[7] J. E. Taylor and J. W. Cahn, "Shape accommodation of a rotating embedded crystal via a new variational formulation," Interfaces and Free Boundaries, vol. 9, no. 4, p. 493, 2007.

[8] H. Zhang, "Atomistic simulation of sliding of [1010] tilt grain boundaries in Mg," Journal of Materials Research, vol. 24, pp. 3446-3453, Jan. 2011.

[9] Z. Trautt, A. Adland, A. Karma, and Y. Mishin, "Coupled motion of asymmetrical tilt grain boundaries: Molecular dynamics and phase field crystal simulations," Acta Materialia, vol. 60, pp. 6528-6546, Nov. 2012.

[10] J. K. Chen, G. Chen, and W. T. Reynolds, "Interfacial structure and growth mechanisms of lath-shaped precipitates in Ni-45 wt Cr," Philosophical Magazine A, vol. 78, pp. 405-422, Aug. 1998.

[11] I. J. Beyerlein, J. R. Mayeur, S. Zheng, N. A. Mara, J. Wang, and A. Misra, "Emergence of stable interfaces under extreme plastic deformation.," Proceedings of the National Academy of Sciences of the United States of America, vol. 111, pp. 4386-4390, Mar. 2014.

[12] G. Wulff, "Zur Frage der Geschwindigkeit des Wachsthums und der Auflösung der Krystallflächen," Zeitschrift für Kristallographie, vol. 34, pp. 449-530, Jan. 1901.

[13] J. Cahn and D. Hoffman, "A vector thermodynamics for anisotropic surfacesII. Curved and faceted surfaces," Acta Metallurgica, vol. 22, pp. 1205-1214, Oct. 1974.

[14] J. E. Taylor, "Crystalline variational problems," Bulletin of the American Mathematical Society, vol. 84, pp. 568-589, July 1978

[15] I. Fonseca, "The Wulff Theorem Revisited," Proceedings of the Royal Society A: Mathematical, Physical and Engineering Sciences, vol. 432, pp. 125-145, Jan. 1991.

[16] C. Herring, "Some Theorems on the Free Energies of Crystal Surfaces," Physical Review, vol. 82, pp. 87-93, Apr. 1951.

[17] B. Dacorogna, Direct methods in the calculus of variations, vol. 78 of Applied Mathematical Sciences. Berlin: SpringerVerlag, 1989.

[18] S. Müller, "Variational models for microstructure and phase transitions," in Calculus of variations and geometric evolution problems (F. Bethuel et al., eds.), Springer Lecture Notes in Math. 1713, pp. 85-210, Springer-Verlag, 1999.

[19] J. W. Cahn and W. C. Carter, "Crystal shapes and phase equilibria: A common mathematical basis," Metallurgical and Materials Transactions A, vol. 27, pp. 1431-1440, June 1996.

[20] J. E. Taylor and J. W. Cahn, "Diffuse interfaces with sharp corners and facets: Phase field models with strongly anisotropic surfaces," Physica D: Nonlinear Phenomena, vol. 112, pp. 381-411, Feb. 1998.

[21] W. K. Allard, "On the First Variation of a Varifold," Annals of Mathematics, vol. 95, no. 3, pp. 417-491, 1972.

[22] F. J. Almgren, Existence and Regularity Almost Everywhere of Solutions to Elliptic Variational Problems with Constraints. American Mathematical Soc., 1976.

[23] J. W. Cahn, "Transitions and phase equilibria among grain boundary structures," Le Journal de Physique Colloques, vol. 43, pp. C6-199-C6-213, Dec. 1982.

[24] J. Hannon, M. Marcus, and V. J. Mizel, "A Variational Problem Modelling Behavior of Unorthodox Silicon Crystals," ESAIM: Control, Optimisation and Calculus of Variations, vol. 9, pp. 145-149, Sept. 2003. 
[25] A. Figalli and F. Maggi, "On the shape of liquid drops and crystals in the small mass regime," Arch. Ration. Mech. Anal., vol. 201, no. 1, pp. 143-207, 2011.

[26] G. Bellettini, V. Caselles, A. Chambolle, and M. Novaga, "The volume preserving crystalline mean curvature flow of convex sets in $\mathbb{R}^{N}$," J. Math. Pures Appl. (9), vol. 92, no. 5, pp. 499-527, 2009.

[27] N. Cabrera, "The equilibrium of crystal surfaces," Surface Science, vol. 2, pp. 320-345, Jan. 1964.

[28] U. Wolf, F. Ernst, T. Muschik, M. W. Finnis, and H. F. Fischmeister, "The influence of grain boundary inclination on the structure and energy of $\sigma=3$ grain boundaries in copper," Philosophical Magazine A, vol. 66, pp. 991-1016, Dec. 1992.

[29] W. Read and W. Shockley, "Dislocation models of crystal grain boundaries," Physical review, vol. 78, no. 3, p. $275,1950$.

[30] J. H. van der Merwe, "On the Stresses and Energies associated with Inter-Crystalline Boundaries," Proceedings of the Physical Society. Section A, vol. 63, pp. 616-637, June 1950.

[31] F. Frank, "The resultant content of dislocations in an arbitrary intercrystalline boundary," in Symposium on The Plastic Deformation of Crystalline Solids, Mellon Institute, Pittsburgh, vol. 150, 1950.

[32] B. A. Bilby, "Report of the Conference on Defects in Crystalline Solids," Tech. Rep. 12, Physical Society, Dec. 1954.

[33] W. Bollmann, Crystal defects and crystalline interfaces. Springer-Verlag, 1970.

[34] N. H. Fletcher, "Crystal interfaces," Journal of Applied Physics, vol. 35, no. 1, pp. 234-240, 1964.

[35] J. Li, "Disclination model of high angle grain boundaries," Surface Science, vol. 31, pp. 12-26, June 1972.

[36] V. V. Bulatov, B. W. Reed, and M. Kumar, "Grain boundary energy function for fcc metals," Acta Materialia, vol. 65, pp. 161-175, Feb. 2014.

[37] B. Runnels, I. J. Beyerlein, S. Conti, and M. Ortiz, "A geometry-based model for the energy of grain boundaries," JMPS (in review), 2015.

[38] L. Ambrosio, N. Fusco, and D. Pallara, Functions of Bounded Variation and Free Discontinuity Problems. Mathematical Monographs, Oxford University Press, 2000.

[39] S. Conti, A. DeSimone, and G. Dolzmann, "Soft elastic response of stretched sheets of nematic elastomers: a numerical study," J. Mech. Phys. Solids, vol. 50, pp. 1431-1451, 2002.

[40] S. Conti, P. Hauret, and M. Ortiz, "Concurrent multiscale computing of deformation microstructure by relaxation and local enrichment with application to single-crystal plasticity," Multiscale Modeling and Simulation, vol. 6, pp. 135-157, 2007.

[41] R. T. Rockafellar, Convex analysis. Princeton Landmarks in Mathematics and Physics Series, Princeton University Press, 1997.

[42] S. Luckhaus and L. Mugnai, "On a mesoscopic many-body Hamiltonian describing elastic shears and dislocations," Continuum Mechanics And Thermodynamics, vol. 22, pp. 251-290, 2010.

[43] S. Luckhaus and J. Wohlgemuth, "Study of a model for reference-free plasticity," preprint arXiv.org 1408.1355, Aug. 2014.

[44] A. P. Sutton and R. Balluffi, Interfaces in crystalline materials. Clarendon Press, 1995.

[45] D. Wolf, "Structure-energy correlation for grain boundaries in F.C.C. metalsIII. Symmetrical tilt boundaries," Acta Metallurgica et Materialia, vol. 38, pp. 781-790, May 1990.

[46] D. Wolf, "Correlation between the energy and structure of grain boundaries in b.c.c. metals. II. Symmetrical tilt boundaries," Philosophical Magazine A, vol. 62, pp. 447-464, Oct. 1990.

[47] D. Wolf, "Structure-energy correlation for grain boundaries in F.C.C. metals I: Boundaries on the (111) and (100) planes," Acta Metallurgica, vol. 37, pp. 1983-1993, July 1989.

[48] D. Wolf, "Structure-energy correlation for grain boundaries in f.c.c. metals IV: Asymmetrical twist (general) boundaries," Acta Metallurgica et Materialia, vol. 38, pp. 791-798, May 1990.

[49] D. Wolf, "Correlation between the energy and structure of grain boundaries in b.c.c. metals I. Symmetrical boundaries on the (110) and (100) planes," Philosophical Magazine Part B, vol. 59, pp. 667-680, June 1989.

[50] D. Wolf, "Structure and energy of general grain boundaries in bcc metals," Journal of Applied Physics, vol. 69, p. 185, Jan. 1991.

[51] M. A. Tschopp and D. L. McDowell, "Structures and energies of $\Sigma 3$ asymmetric tilt grain boundaries in copper and aluminium," Philosophical Magazine, vol. 87, pp. 3147-3173, Aug. 2007.

[52] T. Hsieh and R. Balluffi, "Observations of roughening/de-faceting phase transitions in grain boundaries," Acta Metallurgica, vol. 37, pp. 2133-2139, Aug. 1989

[53] C. Schmidt, M. W. Finnis, F. Ernst, and V. Vitek, "Theoretical and experimental investigations of structures and energies of $\Sigma=3$, [112] tilt grain boundaries in copper," Philosophical Magazine A, vol. 77, pp. 1161-1184, May 1998. 


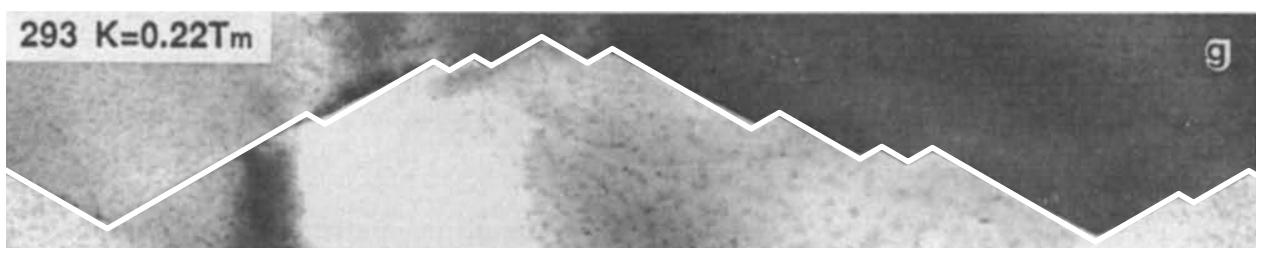

(a) Faceting in $\mathrm{Au}[52$, Figure 7]. Overlay is a trace of the facet pattern using relaxation method-predicted facets.

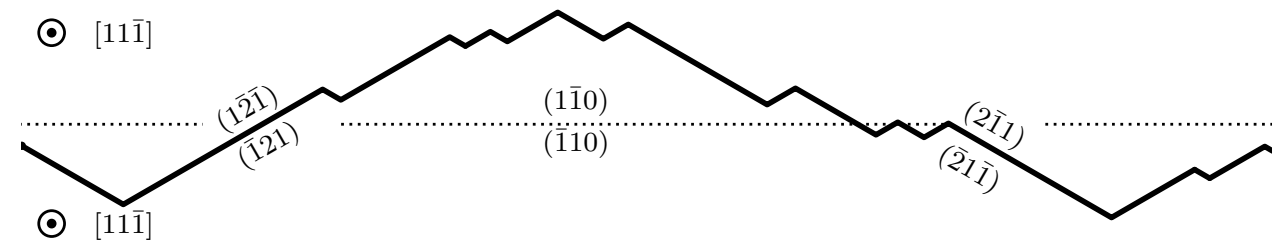

(b) Average ("prescribed") boundary plane and the predicted facet planes

Figure 13: Comparison of experimentally observed faceting in a $\{111\}\langle 112\rangle$ ATGB with the relaxation method's predicted facet pattern.

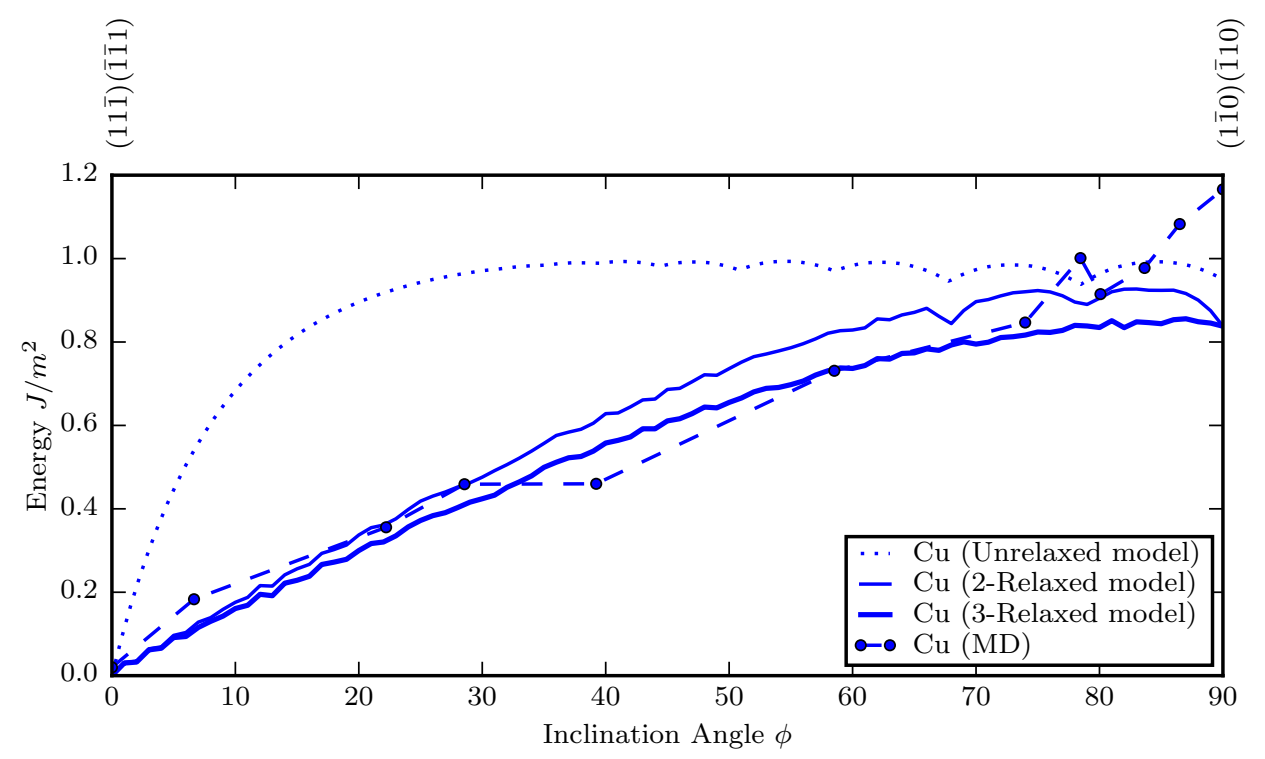

Figure 14: Model prediction of energy for $\{111\}\langle 112\rangle$ ATGBs in copper. Results are presented for the unrelaxed model (dash), relaxed using 2-facets (thin), relaxed using 3-facets (thick). MD results computed with an EAM potential are included for comparison (dot-dash). MD data from [53] 


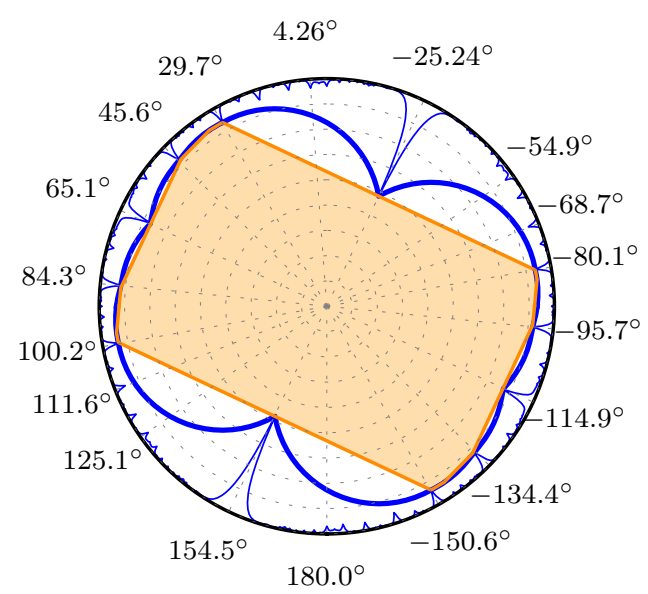

(a) $\sigma=k_{B} T / w_{0}=0.10$

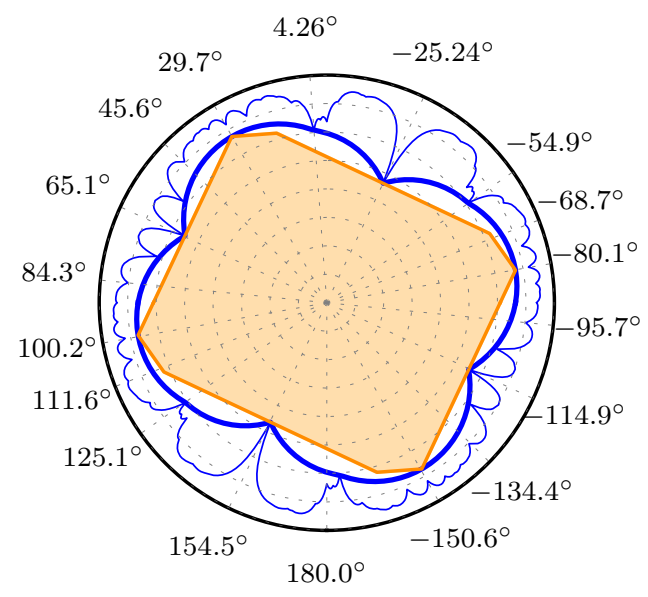

(c) $\sigma=k_{B} T / w_{0}=0.20$

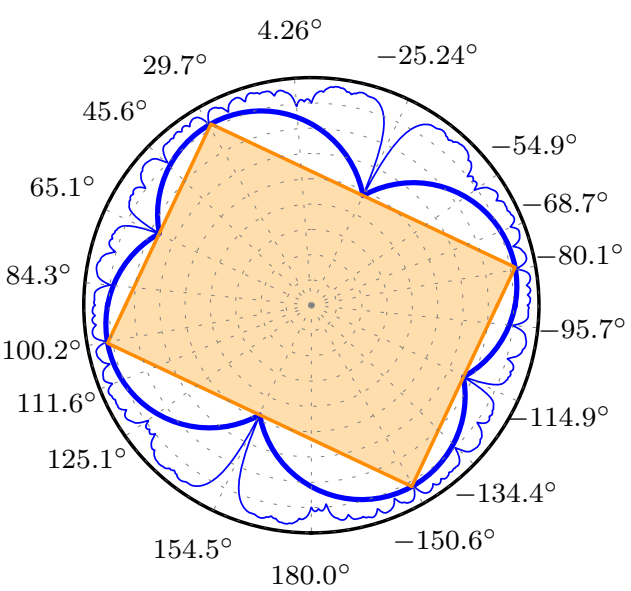

(b) $\sigma=k_{B} T / w_{0}=0.15$

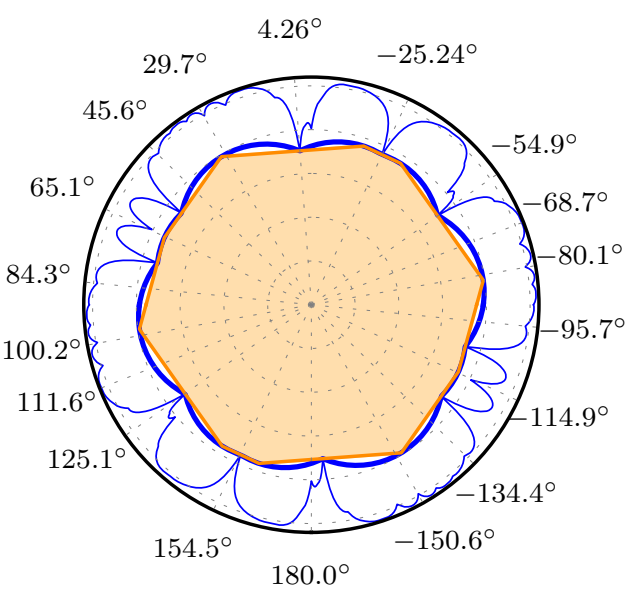

(d) $\sigma=k_{B} T / w_{0}=0.25$

Figure 15: Predicted evolution of the unrelaxed energy surface (thin line) and the relaxed energy surface (thick line) with the temperature $\sigma$. The crystal of the energy surface (i.e. the Wulff shape) is the shaded region. 


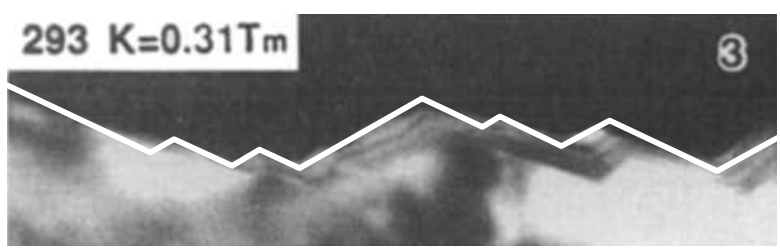

(a) Observed faceting at 293K, with overlay corresponding to facets for $\sigma=0.2,0.25$

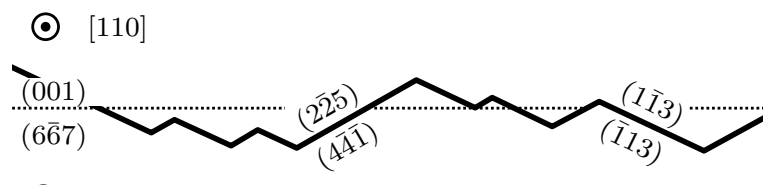

○ $[110]$

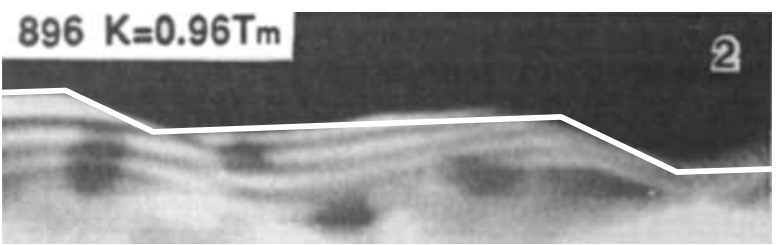

(b) Observed faceting at $896 \mathrm{~K}$, with overlay corresponding to facets for $\sigma=0.1,0.15$

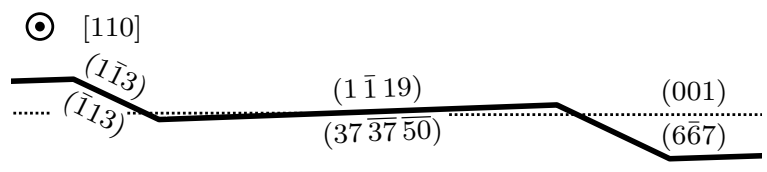

$\odot$ [110]

(c) Facet planes at $293 \mathrm{~K}$

(d) Facet planes at $896 \mathrm{~K}$

Figure 16: Experimentally observed faceting in $\mathrm{Al}$ changing with temperature [52, Figure 8a]. The superimposed white lines in the figures are an approximation of the interface using facet slopes predicted by the model. 